\title{
Modelling and analysis of coupled flap-lag-torsion vibration characteristics for helicopter rotor blades
}

Proc IMechE Part G: $\mathrm{J}$ Aerospace Engineering 000(00):1-13 (C) The Author(s) 2016 Reprints and permission: sagepub.co.uk/journalsPermissions.nav DOI:doi number http://mms.sagepub.com

\author{
Alejandro Castillo Pardo, Ioannis Goulos, and Vassilios Pachidis \\ Propulsion Engineering Centre, Cranfield University, UK
}

\begin{abstract}
This paper presents the development of a mathematical approach targeting the modelling and analysis of coupled flap-lagtorsion vibration characteristics of non-uniform continuous rotor blades. The proposed method is based on the deployment of Lagrange's equation of motion to the three-dimensional kinematics of rotor blades. Modal properties derived from classical beam and torsion theories are utilized as assumed deformation functions. The formulation, valid for hingeless, freely-hinged, and spring-hinged articulated rotor blades, is reduced to a set of closed form integral expressions. Numerical predictions for mode shapes and natural frequencies are compared with experimental measurements, nonlinear finite element analyses, and multi-body dynamics analyses for two small scale hingeless rotor blades. Excellent agreement is observed. The effect of different geometrical parameters on the elastic and inertial coupling is assessed. Additionally, the effect of the inclusion of gyroscopic damping is evaluated. The proposed method, able to estimate the 7 first coupled modes of vibration in a fraction of a second, exhibits an excellent numerical stability. It constitutes a computationally efficient alternative to multi-body dynamics and finite element analysis for the integration of rotor blade flexible modelling into a wider comprehensive rotorcraft tool.
\end{abstract}

Keywords

Structural dynamics, vibrations, natural frequencies, coupling, rotor blades, rotorcraft

\section{Introduction}

\subsection{Background}

A significant evolution in structural modelling of rotor blades has taken place from the elementary beam theories to more refined modern finite element analysis (FEA) theories. Classical beam theory was deployed as a first attempt to model the structural dynamics of rotor blades. It comprised Euler-Bernoulli beam theory for the extension and bending and SaintVenant's theory for torsion. Coupling between bending, extension and torsion was not modelled. Timoshenko (Ref. 1) enhanced the classical theory accounting for transverse shear deformation and cross-sectional rotary inertia. Vlasov (Ref.

*Corresponding author; e-mail: a.castillopardo@ cranfield.ac.uk 
2) incorporated torsional warping restraint to the previous theory. However, the rotation of the blade was not accounted for and the degrees of freedom (DOFs) were treated individually, therefore these theories were unsuitable for rotor blades.

During the late 1950s Houbolt and Brooks (Ref. 3) formulated the differential equations of motion for the coupled flapwise/chordwise bending and torsion of a twisted non-uniform rotor blade subjected to linear aerodynamic loads. The theory was based on the classical engineering beam theory. It was reduced to a set of linear second order ordinary differential equations neglecting shear deformation, rotary inertia and other nonlinear terms of secondary nature. Although this theory clearly omitted the effect of nonlinear terms, it provided physical insight of the problem and constituted the basis for many subsequent analyses where approximate solutions of the set of equations were obtained.

A nonlinear set of equations of motion was derived by Hodges and Dowell (Ref. 4) during the 1970s for the coupled flapwise/chordwise bending and torsion of rotor blades. A truncation scheme which reduced the rotor blade to a long, straight, slender, homogeneous, and isotropic beam undergoing moderate deflections was deployed to simplify the equations. Two methods were used to derive the theory: Hamilton's principle and Newtonian methods. The strain-displacement relations were derived from the geometrically exact transformation between the deformed and undeformed system. Although the use of ordering schemes reduced the non-linearities considerably, several nonlinear structural and inertial coupling terms still appeared in the final set of equations. These effectively represented the nonlinear coupling between axial extension, flapwise/chordwise bending, and torsion. The relevance of these nonlinear coupling terms for aeroelastic applications was later emphasized in (Ref. 5).

A series of new ordering schemes were developed in the 1970s and 1980s. These theories were based on small-strain approximations. These did not explicitly limit the displacements of the reference line and the rotations of the cross-section caused by deformations. This led to a generalization of the often called "geometrically exact theories". A representative example of this type of theory is the study of the kinematics of elastic motion of Bernoulli-Euler beams subjected to large deflections carried out by Hodges et al. (Ref. 6). As a result, nonlinear expressions relating the orientation of the deformed cross-section, torsion, local bending curvature, angular velocity, and virtual rotation with deformation variables were obtained. A posterior study carried out by the same authors (Ref. 7) extended the methodology of Ref. 6 to the dynamic analysis of pretwisted rotating beams.

Berdichevsky (Ref. 8) proposed the variational-asymptotic method (VAM). VAM divides the 3D geometrically nonlinear elastic analysis into two different analyses: a nonlinear 1D analysis and a 2D analysis. Energy functionals or variational principles are asymptotically expanded as functions of small parameters. Hodges et al. (Refs. 9, 10) extrapolated VAM to beams, plates and shells made of non-homogeneous composite materials. Although computationally more efficient than FEM, its computational overhead and the large amount of data required makes this tool not suitable for preliminary design.

Most of the aforementioned references are focused on the development of the kinematic expressions of a beam element However, the equations of motion obtained have no analytical solutions. In most cases numerical methods methods were developed to estimate the response of the coupled system or simplified uncoupled cases.

Murthy (Ref. 11) deployed the Transmission Matrix (MT) to estimate the solution of the linear differential equations of Houbolt and Brooks (Ref. 3) for a twisted non-uniform blade. The TM method carries out a transformation of the differential equation of motion into first order differential equations using a state vector. Once the transformation was performed, modal characteristics were obtained for a particular set of boundary conditions. Accurate results were obtained for combined flapwise bending/chordwise bending/torsion, flapwise bending/chordwise bending, and flapwise bending/torsion.

Surace et al. (Ref. 12) used Green functions to calculate the coupled vibration characteristics for a rotating nonuniform pretwisted blade. Specific Green functions were imposed to satisfy the boundary conditions. Weighting matrices were required for numerical integration and differentiation. Structural influence functions are only available for hingeless beams. Therefore, they need to be derived for freely-hinged and spring-hinged articulated rotor blades. 


\subsection{Minimum potential energy methods}

Both numerical methods presented above are based on the numerical evaluation of Houbolt and Brooks equations. However, alternative approaches based Lagrange's method, Rayleigh's method, and Rayleigh-Ritz procedures were have also been deployed in the existing literature.

Fasana and Marchesiello (Ref. 13) used Rayleigh-Ritz method to study the modal characteristics of sandwich beams with constrained viscoelastic layer. 20 simple polynomial expressions were utilized as assumed deformation functions. Up to 80 polynomial expressions were deployed to evaluate the sensibility of the results on the number of assumed deformation functions. No significant variation in the estimated results were found. The first four predicted bending natural frequencies were compared with other numerical methods estimations. Good agreement was found for the lowest natural modes, nevertheless, for higher modes discrepancies between models increased considerably.

Hodges (Ref. 14) deployed a modified Ritz method to analyse non-uniform rotating beams. Radial discontinuities in bending stiffness and mass per unit length were included. The structural properties discontinuity required the use of $M+1$ continuous segments with $M$ discontinuities in structural properties. Hodges replaced standard polynomial expressions with power series of $\mathrm{N}$ terms for the formulation of the assumed deformation functions within each discrete segment. Additionally, geometric continuity was obtained at the extremes of each segment imposing boundary conditions. The relevance of the set of deformation functions assumed was emphasized by the author.

Goulos et al. (Ref. 15) deployed Lagrange's method to estimate the modal characteristics of non-uniform rotating helicopter blades. Mode shapes derived from classical beam and torsional theories were utilized as assumed deformation functions. The employed functions satisfied the structural boundary conditions for a series of hub supports. These were linearly independent and close to the actual deformation mode. The latter reduced the number of required assumed modes to attain convergence. As a result, computational times were reduced considerably leading to a computationally efficient method. The method proposed described in Ref. 15 treated flapwise, chordwise displacements and torsion independently, i.e. uncoupled. However, very good agreement with the experimental measurements, FEA, and multi-body dynamics analyses was observed. Subsequently, Goulos et al. 16-18 applied the proposed structural model to real-time helicopter flight dynamic integrating the proposed method into a helicopter comprehensive code.

\subsection{Scope of present work}

Within this context it is clear the absence of methods able to rapidly estimate the coupled vibration characteristics of helicopter rotor blades. The potential method has to be able to solve the aforementioned weaknesses: model coupling between DOFs, be expressed in closed form expressions readily available for any type of hub support, require a limited amount of information, and deploy a reduce number of assumed deformation functions to enhance the computational efficiency.

This paper presents a mathematical formulation that meets the previous requirements. It is capable of modelling the coupled flap/lag bending and torsion natural frequencies and mode shapes of non-uniform rotor blades. The method is based on the deployment of Lagrange's equation of motion to the three-dimensional kinematics of rotor blades. It uses mode shapes derived from classical beam and torsional theories as assumed deformation functions. These better approximate the actual deformation of the blade accelerating the convergence of the method. As a result, the computational efficiency of the method is considerably improved.

Closed form integral expressions for the kinetic and potential energies are formulated. Lagrange's equation of motion is deployed to obtain the dynamic system's set of second-order ordinary differential equations. The latter is transformed into a quadratic eigenvalue problem. Its solution comprises natural frequencies and mode shapes of the non-uniform rotating blade. Different sources of coupling between modes of vibration are identified. An extensive analysis of the effect of the inclusion of gyroscopic damping on the estimated modal characteristics is carried out. 


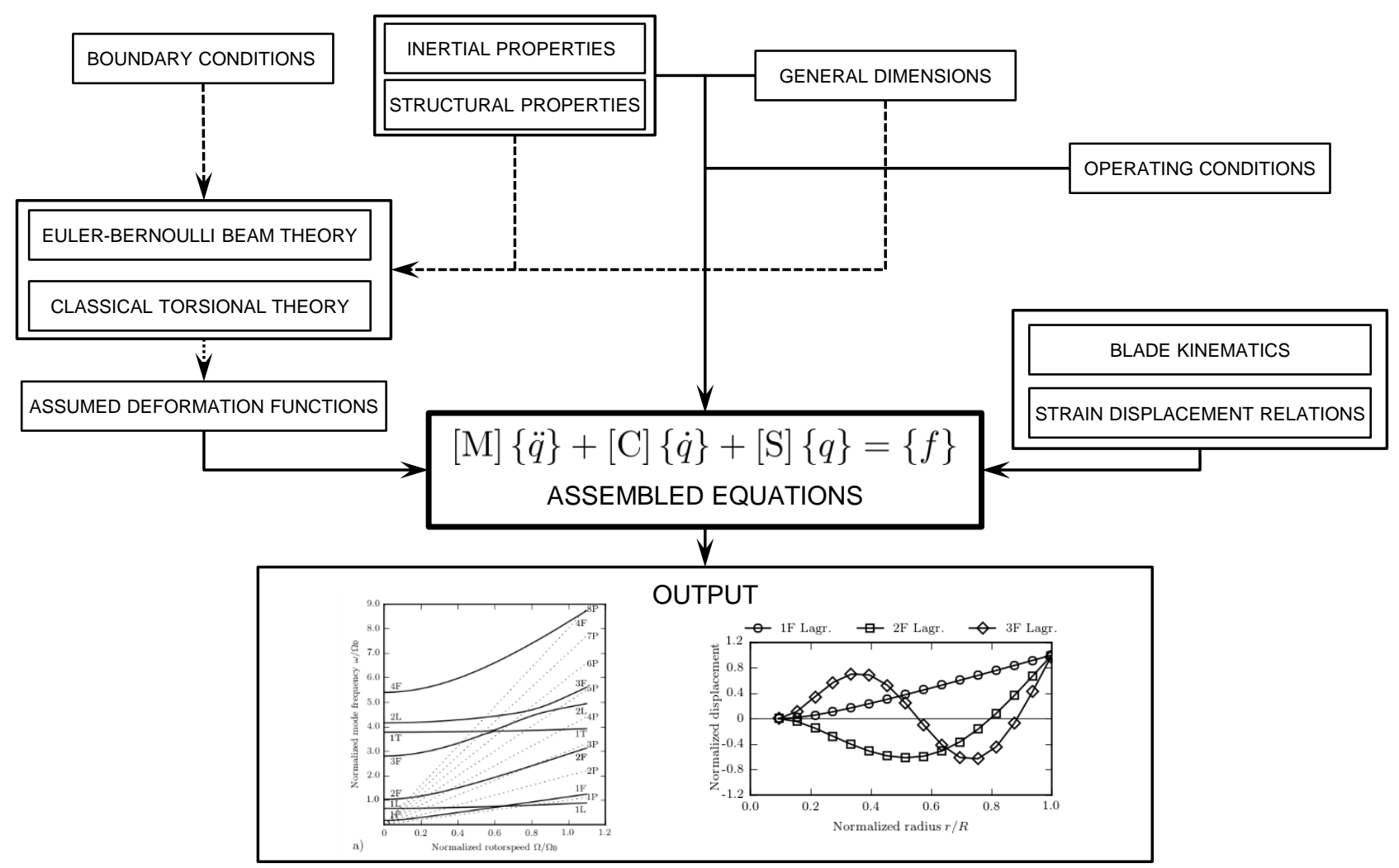

Fig. 1. Model flowchart.

Figure 1 summarises the main features of the proposed methodology. The classical beam and torsional theories are utilised to obtain the assumed mode shapes. These require inertial properties, structural properties, general dimensions and structural boundary conditions as inputs. The derived assumed mode shapes are deployed along with blade properties, operating conditions, blade kinematics and strain displacement relations to fill the mass, gyroscopic, and stiffness matrix. These form the quadratic eigenvalue problem solved to obtain the final natural frequencies and mode shapes.

The proposed method constitutes a computationally efficient alternative to multi-body dynamics and FEA for the structural treatment of rotor blades. The limited information required by the model makes it a suitable tool for structural studies during preliminary design phases. The proposed method can be deployed along with any inflow, and aerodynamic blade models to carry out aeroelastic analyses in the time domain. The further integration of the mentioned aeroelastic model along with a flight-dynamic model sets up the basis of a comprehensive rotorcraft code. Its excellent computational performance and ease of implementation present this method as an optimal choice for its integration into a comprehensive rotorcraft code.

\section{Mathematical formulation}

\subsection{Derivation of assumed deformation functions}

Lagrange's equation of motion requires the formulation of the kinetic and strain energies as functions of generalized coordinates and assumed deformation functions. An effective modal coordinate $x=r-e R, x \in(0, L)$ is defined. Where $r \in(e R, R)$ is the local blade radius, $e$ is the ratio between the hinge/root offset from the center of the rotor hub and the blade tip radius $R$, and $L=R(1-e)$ is the actual blade length. 
The classical Bernoulli-Euler beam theory (Eq. (1a)) is the governing equation for the transverse displacement $w(x, t)$ of a non-rotating beam with variable bending stiffness $E I(x)$, mass per unit length $\rho A(x)$, and load per unit length $P(x, t)$. The classical torsional theory (Eq. (1b)) is the governing equation for the torsion angle $\theta(x, t)$ for a non-rotating beam with variable torsional rigidity $G J(x)$, polar moment of inertia $I_{p}(x)$, and torsional moment loads per unit length $M(x, t)$.

$$
\begin{aligned}
& \frac{\partial^{2}}{\partial x^{2}}\left(E I(x) \frac{\partial^{2} w(x, t)}{\partial x^{2}}\right)+\rho A(x) \frac{\partial^{2} w(x, t)}{\partial t^{2}}=P(x, t) \\
& \frac{\partial}{\partial x}\left(G J(x) \frac{\partial \theta(x, t)}{\partial x}\right)+M(x, t)=I_{p}(x) \frac{\partial^{2} \theta(x, t)}{\partial t^{2}}
\end{aligned}
$$

Uniform structural properties and zero external forcing are assumed in Eqs. (1a) and (1b). Separation of variables is applied to the simplified equations leading to the spatial solutions for transverse displacement $\Phi(x)$ and torsion angle $\Theta(x)$.

$$
\begin{aligned}
& \Phi(x)=A_{\Phi} \sin \beta x+B_{\Phi} \cos \beta x+C_{\Phi} \sinh \beta x+D_{\Phi} \cosh \beta x \\
& \Theta(x)=A_{\Theta} \cos \gamma x+B_{\Theta} \sin \gamma x
\end{aligned}
$$

Where $\beta$ and $\gamma$ are the frequency parameters for transverse displacement and torsional deformation respectively. $A_{\Phi}, B_{\Phi}$, $C_{\Phi}, D_{\Phi}, A_{\Theta}$, and $B_{\Theta}$ are constants of integration determined through application of boundary conditions. Goulos $e t$ al. (Ref. 15) provided closed form expressions for the ratios between the constants of integration and frequency parameters for hingeless, freely-hinged articulated, and spring-hinged articulated rotor blades, and rotor blade pitch-control system with finite and infinite torsional stiffness. The application of this procedure to flap, lag, and torsion results in the derivation of $N$ assumed deformation functions per DOF. The deformation functions, calculated for a non-rotating beam with constant properties, satisfy the structural boundary conditions and exhibit orthogonality between each other. The deformation functions are utilised to better approximate the actual mode shapes for rotating non-uniform rotor blades with coupled modes of vibration. These functions are expressed in vector form as $\left\{\psi^{\phi}(x)\right\}=\left[\Theta_{i}(x), i=1, \ldots N\right]^{T},\left\{\psi^{v}(x)\right\}=$ $\left[\Phi_{i}^{v}(x), i=1, \ldots N\right]^{T}$, and $\left\{\psi^{w}(x)\right\}=\left[\Phi_{i}^{w}(x), i=1, \ldots N\right]^{T}$ for torsion, lag, and flap respectively. The assumed deformation function subscript $i \in[1, \ldots N]$ refers to the $i$ th assumed deformation function. Similarly, the 1 st and 2 nd spatial derivatives can be obtained in closed form expressions. Thus, numerical errors associated with numerical derivation are not generated. These derivatives are expressed as the following vectors $\left\{\psi^{\prime r}(x)\right\}$ and $\left\{\psi^{\prime \prime r}(x)\right\}$ where the DOF superscript $r \in[\phi, v, w]$ refers to the DOF associated with the vector: $\phi, v$, and $w$ for torsion, lag, and flap respectively.

\subsection{Lagrangian formulation for rotor blade dynamics}

Lagrange's equation of motion The proposed formulation accounts for inertial and elastic coupling between torsional deformation, chordwise displacement and flapwise displacement. Lagrange's equation of motion, defined in Eq. (3), is applied to torsion, lag, and flap independently. In Eq. (3), $T$ and $U$ are the kinetic and strain energies of the complete system, $Q_{i}^{r}$ is the generalized external force/moment and $q_{i}^{r}(t)$ is the time-dependent generalized coordinate.

$$
\frac{\mathrm{d}}{\mathrm{d} t}\left(\frac{\partial T}{\partial \dot{q}_{i}^{r}}\right)-\frac{\partial T}{\partial q_{i}^{r}}+\frac{\partial U}{\partial q_{i}^{r}}=Q_{i}^{r}, \quad i=1, \ldots N
$$

The generalized coordinates can be expressed as $\left\{q^{r}(t)\right\}=\left[q_{i}^{r}(t), i=1, \ldots N\right]^{T}$, whilst the transverse displacements and torsional deformation as the dot products of the assumed deformation function and the generalized coordinate vectors.

$$
\begin{aligned}
\phi(x, t) & =\left\{\psi^{\phi}(x)\right\} \cdot\left\{q^{\phi}(t)\right\} \\
v(x, t) & =\left\{\psi^{v}(x)\right\} \cdot\left\{q^{v}(t)\right\} \\
w(x, t) & =\left\{\psi^{w}(x)\right\} \cdot\left\{q^{w}(t)\right\}
\end{aligned}
$$

The calculation of the rotor blade modal characteristics does not require the inclusion of external loads in the analysis. Therefore, the calculation of the generalized external force/moment, $Q_{i}^{r}$, is not necessary and is eliminated from Eq. (3). 


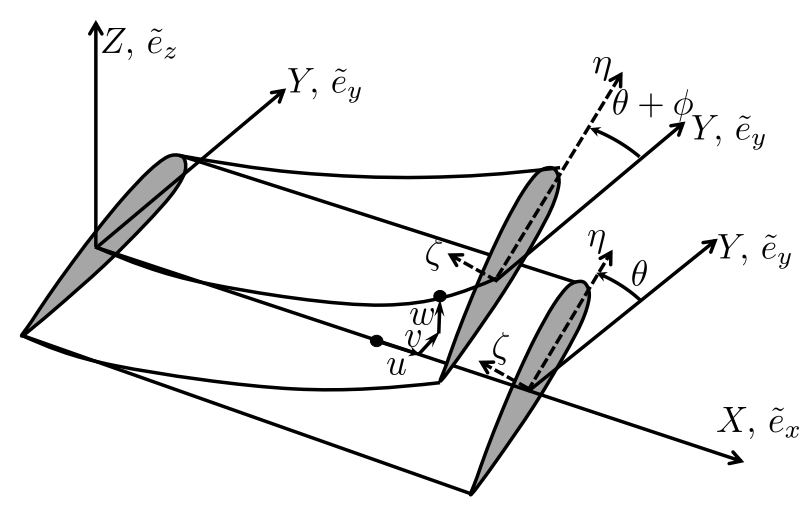

Fig. 2. Cross-section frame of reference.

Frames of reference. Transverse displacements and torsional deformation of the rotor blade are expressed in a frame of reference fixed to the blade with origin at the root/hinge. This reference system, shown in Fig. 2, is non-inertial due to the rotation of the blade about the hub axis and introduces nonlinear phenomena in the analysis. The position vector of an arbitrary point $P$ on the elastic axis before deformation is $\tilde{r}_{0}=\left[\begin{array}{lll}x & 0 & 0\end{array}\right]^{T}$. After the deformation, the same point is located at $\tilde{r}_{1}=\left[\begin{array}{lll}x+u & v & w\end{array}\right]^{T}$ where $u, v$ and $w$ are the axial, chordwise and flapwise displacements of $P$ in $\tilde{e}_{x}, \tilde{e}_{y}$ and $\tilde{e}_{z}$ respectively as shown in Fig 2. An extra two-dimensional reference system is defined for the cross-sectional plane, it is based on the major $\eta$ and minor $\zeta$ principal axes with origin at the elastic axis. The angle between the axes $Y$ and $\eta$ prior to deformation is the pretwist angle $\theta$. After the deformation, the angle between $Y$ and $\eta$ becomes $\theta+\phi$, where $\phi$ represents the elastic torsion angle.

Strain Energy Prior to the application of Lagrange's equation of motion, the strain energy is expressed in terms of the generalized coordinates and the assumed deformation functions. Based on the assumption of uniaxial stress and the validity of the Generalized Hooke's law, the strain energy is defined as follows

$$
U=\frac{1}{2} \int_{0}^{L} \iint_{A}\left(E \epsilon_{x x}^{2}+G \epsilon_{x \eta}^{2}+G \epsilon_{x \zeta}^{2}\right) \mathrm{d} \zeta \mathrm{d} \eta \mathrm{d} x+\frac{1}{2}\left(K^{\phi} \phi_{0}^{2}+K^{v} v_{0}^{\prime 2}+K^{w} w_{0}^{\prime 2}\right)
$$

where $\epsilon_{x x}, \epsilon_{x \eta}$, and $\epsilon_{x \zeta}$ are the classical strain components, $E$ and $G$ are the material Young's and shear stress moduli, $\phi_{0}$ is the torsional deformation at the root/hinge, and $v_{0}^{\prime}$ and $w_{0}^{\prime}$ are the linearised chordwise and flapwise angular deformations of the hinge springs. In the case of a spring hinged articulated blade, the last group of terms in Eq. (5) accounts for the extra strain energy due to the presence of discrete springs with $K^{v / w}$ stiffness and/or a pitch-control system with finite torsional stiffness $K^{\phi}$ at the root/hinge location.

A series of assumptions reduces the strain displacements relations developed by Hodges and Dowel (Ref. 4) to the classical strain components $\epsilon_{x x}, \epsilon_{x \eta}, \epsilon_{x \zeta}$ used in this formulation. The assumption of uniaxial stress simplifies the actual blade to a long, straight, slender, homogeneous, and isotropic beam undergoing moderate displacements. For the purpose of this work, the effect of cross-sectional warp is neglected, the expressions are reduced to second order, and the trigonometric functions containing the torsion angle are approximated with Maclaurin series expansions of second order. The resulting strain components are presented in Eq. (6) where ()$^{\prime}$ and ()$^{\prime \prime}$ represent the first and second derivatives with respect to $x$.

$$
\begin{aligned}
\epsilon_{x x}= & u^{\prime}+\frac{v^{\prime 2}}{2}+\frac{w^{\prime 2}}{2}+\left(\eta^{2}+\zeta^{2}\right)\left(\theta^{\prime} \phi^{\prime}+\frac{\phi^{\prime 2}}{2}\right)-\left(v^{\prime \prime}+w^{\prime \prime} \phi-v^{\prime \prime} \frac{\phi^{2}}{2}\right)(\eta \cos \theta-\zeta \sin \theta) \\
& -\left(w^{\prime \prime}-v^{\prime \prime} \phi-w^{\prime \prime} \frac{\phi^{2}}{2}\right)(\eta \sin \theta+\zeta \cos \theta) \\
\epsilon_{x \eta}= & -\zeta \phi^{\prime} \\
\epsilon_{x \zeta}= & \eta \phi^{\prime}
\end{aligned}
$$


The axial displacement $u$ is expressed as a function of the transverse displacements $v$ and $w$, and torsion angle $\phi$ through application of Eq. (7). This equation is an adaptation of the methodology used in Ref. 4.

$$
u^{\prime}=\frac{\int_{x}^{L} m \Omega^{2}(\varrho+e R) \mathrm{d} \varrho}{E A}-\frac{1}{2} v^{\prime 2}-\frac{1}{2} w^{\prime 2}-\frac{J}{A} \theta^{\prime} \phi^{\prime}+e_{A} v^{\prime \prime} \cos \theta+e_{A} w^{\prime \prime} \sin \theta-e_{A} v^{\prime \prime} \phi \sin \theta+e_{A} w^{\prime \prime} \phi \cos \theta
$$

Equation (7) deploys cross-sectional integrals defined in Eq. (8). These integrals, which are functions of the material density $\rho$ and the cross-sectional major $\eta$ and minor $\zeta$ axes coordinates, are evaluated only over the area of the blade cross-section structurally effective. $A$ is the cross-sectional area carrying tension, $m$ is the blade mass per unit length, $e_{m}$ and $e_{A}$ are the offsets of the mass and area centroids from the elastic axis respectively, $I_{y}$ and $I_{z}$ are the flapwise and chordwise area moments of inertia respectively, $I_{1}$ and $I_{2}$ are the flapwise and chordwise mass moments of inertia respectively, $J$ is the cross-sectional polar moment of inertia, $I_{p}$ is the cross-sectional mass polar moment of inertia, and $B_{1}^{*}$ and $B_{2}^{*}$ are section constants equivalent to those of Refs. 3 and 4 . It is noted that in order to not overcomplicate the formulation with low order of magnitude terms, the cross-section is assumed to be symmetric about the major axis $\eta$. This essentially eliminates the cross-sectional integrals of odd-degree $\zeta$ polynomials. In Eq. (7) the first three terms have the most relevant effect on the axial displacement. The first term is directly associated with centrifugal stiffening while the following two terms are associated with the effect of transverse dislocations on the axial extension.

$$
\begin{array}{lll}
A=\iint_{A} \mathrm{~d} \zeta \mathrm{d} \eta & m=\iint_{A} \rho \mathrm{d} \zeta \mathrm{d} \eta & m e_{M}=\iint_{A} \rho \eta \mathrm{d} \zeta \mathrm{d} \eta \\
B_{1}^{*}=\iint_{A}\left(\eta^{2}+\zeta^{2}\right)^{2} \mathrm{~d} \zeta \mathrm{d} \eta & B_{2}^{*}=\iint_{A} \eta\left(\eta^{2}+\zeta^{2}\right) \mathrm{d} \zeta \mathrm{d} \eta & A e_{A}=\iint_{A} \eta \mathrm{d} \zeta \mathrm{d} \eta \\
I_{y}=\iint_{A} \zeta^{2} \mathrm{~d} \zeta \mathrm{d} \eta & I_{z}=\iint_{A} \eta^{2} \mathrm{~d} \zeta \mathrm{d} \eta & J=\iint_{A}\left(\eta^{2}+\zeta^{2}\right) \mathrm{d} \zeta \mathrm{d} \eta \\
I_{1}=\iint_{A} \rho \zeta^{2} \mathrm{~d} \zeta \mathrm{d} \eta & I_{2}=\iint_{A} \rho \eta^{2} \mathrm{~d} \zeta \mathrm{d} \eta & I_{p}=\iint_{A} \rho\left(\eta^{2}+\zeta^{2}\right) \mathrm{d} \zeta \mathrm{d} \eta
\end{array}
$$

The final strain energy expression is derived inserting Eqs. (6), and (7) in Eq. (5) and expressing the transverse displacement and torsional deformation as the dot products of the assumed deformation function and the generalized coordinate vectors (Eq. (4)). The proposed approach targets the rapid estimation of helicopter rotor blade vibration characteristics. In order to accomplish the computational efficiency and make it suitable for preliminary design, the products of $v, w$, and/or $\phi$ of third degree or higher are neglected. Additionally, flap and lead-lag hinges are assumed to be coincident and their position determines the actual length of the blade $L$.

Kinetic energy The kinetic energy is defined in Eq. (9) as the volume integral of the density times the dot product of the absolute velocity vector $\tilde{V}$ of an arbitrary point $P$. The latter is, defined in Eq. (10), comprises the following terms expressed in the blade fixed reference frame: linear velocity of the root/hinge $\tilde{V}_{0}$, deformed position vector $\tilde{r}$, time derivative of the deformation position vector $\frac{\partial \tilde{r}}{\partial t}$ and the angular velocity vector $\tilde{\Omega}$. $\vec{V}_{0}$ and $\tilde{\Omega}$ are defined in Eqs. (11) and (12) in terms of the rotorspeed $\Omega$, root/hinge offset $e R$, and pre-cone angle $\beta$.

$$
\begin{aligned}
T & =\frac{1}{2} \int_{0}^{L} \iint_{A} \rho \tilde{V} \cdot \tilde{V} \mathrm{~d} \zeta \mathrm{d} \eta \mathrm{d} x \\
\tilde{V} & =\tilde{V}_{0}+\frac{\partial \tilde{r}}{\partial t}+\tilde{\Omega} \times \tilde{r} \\
\tilde{V}_{0} & =\left[\begin{array}{lll}
0 & \Omega e R & 0
\end{array}\right]^{T} \\
\tilde{\Omega} & =\left[\begin{array}{lll}
\Omega \sin \beta & 0 & \Omega \cos \beta
\end{array}\right]^{T}
\end{aligned}
$$

The deformed position vector $\tilde{r}$ of an arbitrary point $P$ is based on the kinematic expressions derived by Hodges and Dowel (Ref. 4) for a long, straight beam undergoing moderate displacements. Warping is neglected and the trigonometric functions containing the torsion angle are approximated using second order Maclaurin series expansions. The resulting position vector $\tilde{r}$ is shown in Eq. (13). Once again, the products of $v, w$, and/or $\phi$ of third degree or higher are neglected, and the flap and lag hinges are assumed to be coincident. However, the pre-cone angle's trigonometric functions are approximated using Maclaurin series expansions of first order. 


$$
\tilde{r}=\left\{\begin{array}{c}
x+u-\left(v^{\prime}+w^{\prime} \phi\right)[\eta \cos \theta-\zeta \sin \theta]-\left(w^{\prime}-v^{\prime} \phi\right)[\eta \sin \theta+\zeta \cos \theta] \\
v+[\eta \cos \theta-\zeta \sin \theta]-\phi[\eta \sin \theta+\zeta \cos \theta]-\frac{\phi^{2}}{2}[\eta \cos \theta-\zeta \sin \theta] \\
w+[\eta \sin \theta+\zeta \cos \theta]+\phi[\eta \cos \theta-\zeta \sin \theta]-\frac{\phi^{2}}{2}[\eta \sin \theta+\zeta \cos \theta]
\end{array}\right\}
$$

\subsection{Eigenproblem}

Once the strain and kinetic energies have been formulated, the transverse displacements and torsional deformation are substituted by the equivalent products of assumed deformation functions and generalized coordinates (Eq. 4). This results on the final expressions of the kinetic and strain energies. The application of Lagrange's equation of motion (Eq. 3) to the strain and kinetic energies expressions leads to the following second order system of ordinary differential equations

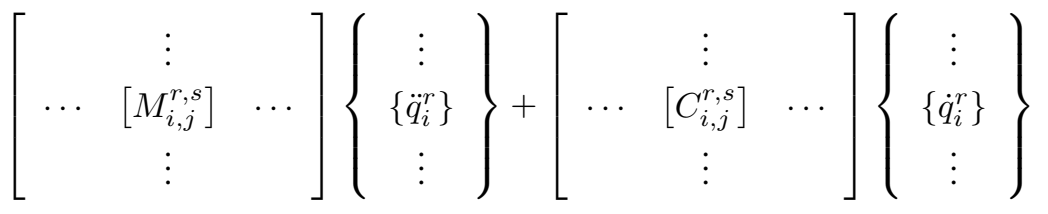

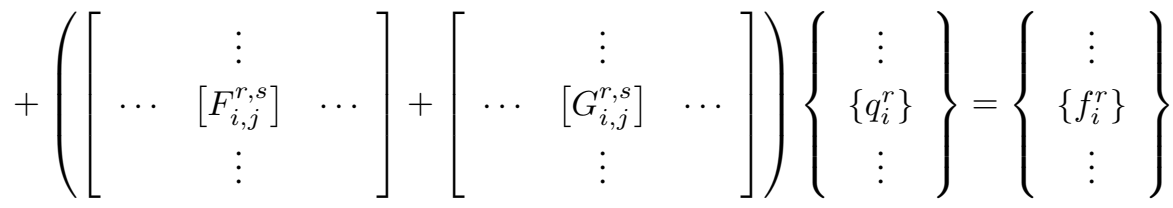

or

$$
[M]\{\ddot{q}\}+[C]\{\dot{q}\}+[S]\{q\}=\{f\}
$$

where $[M],[C]$, and $[S]$ are the mass, damping, and overall stiffness intermodal coupling matrices. The overall stiffness intermodal coupling matrix $[S]$ comprises contributions of two different nature: structural stiffening $[G]$ and centrifugal stiffening $[F] .\{f\}$ is denominated external static loading forcing vector. $\{q\},\{\dot{q}\}$, and $\{\ddot{q}\}$ are the generalized coordinates vector and its first and second time derivatives, respectively. In Eq. (14), the DOF superscripts $r$ and $s$ refer to a partition number (row or column) that corresponds to a DOF ( $\phi$ for torsion, $v$ for lag, and $w$ for flap). It is noted that consecutive integers $(1,2$, and 3) are used as DOF indices in the mathematical implementation of the model. However, the use of $\phi$, $v$, and $w$ simplifies the description. The mode subscripts $i$ and $j$ correspond to row/column indices within each partition matrix. Thus, $M_{i, j}^{r, s}$ gives the mass intermodal coupling coefficient of the $i$ th assumed deformation function of the $r$ DOF with to $j$ th assumed deformation function of the $s$ DOF. It is noted that these coefficients are exclusively functions of $x$.

At this point, each of the above matrices is derived. The mass intermodal coupling matrix $[M]$ is symmetric and positive definite. It is associated with the kinetic energy due to time-variations of the elastic deformation of the blade. The individual components of the $[M]$ matrix are defined as follows:

$$
\begin{aligned}
M_{i, j}^{\phi, \phi} & =\int_{0}^{L} m \int_{0}^{x} \frac{J}{A} \theta^{\prime} \psi_{i}^{\prime} \mathrm{d} \lambda \int_{0}^{x} \frac{J}{A} \theta^{\prime} \psi_{j}^{\prime} \mathrm{d} \lambda \mathrm{d} x+\int_{0}^{L} I_{p} \psi_{i} \psi_{j} \mathrm{~d} x \\
M_{i, j}^{\phi, v} & =M_{j, i}^{v, \phi}=\int_{0}^{L} m\left(-e_{M} \sin \theta \psi_{i} \psi_{j}+\int_{0}^{x} \frac{J}{A} \theta^{\prime} \psi_{i}^{\prime} \mathrm{d} \lambda\left(e_{M} \cos \theta \psi_{j}^{\prime}-\int_{0}^{x} e_{A} \cos \theta \psi_{j}^{\prime \prime} \mathrm{d} \lambda\right)\right) \mathrm{d} x \\
M_{i, j}^{\phi, w} & =M_{j, i}^{w, \phi}=\int_{0}^{L} m\left(e_{M} \cos \theta \psi_{i} \psi_{j}+\int_{0}^{x} \frac{J}{A} \theta^{\prime} \psi_{i}^{\prime} \mathrm{d} \lambda\left(e_{M} \sin \theta \psi_{j}^{\prime}-\int_{0}^{x} e_{A} \sin \theta \psi_{j}^{\prime \prime} \mathrm{d} \lambda\right)\right) \mathrm{d} x \\
M_{i, j}^{v, v} & =\int_{0}^{L} m\left(\psi_{i} \psi_{j}+\int_{0}^{x} e_{A} \psi_{i}^{\prime \prime} \cos \theta \mathrm{d} \lambda \int_{0}^{x} e_{A} \psi_{j}^{\prime \prime} \cos \theta \mathrm{d} \lambda-m e_{M} \cos \theta\left(\psi_{i}^{\prime} \int_{0}^{x} e_{A} \psi_{j}^{\prime \prime} \cos \theta \mathrm{d} \lambda+\right.\right. \\
& \left.\left.\psi_{j}^{\prime} \int_{0}^{x} e_{A} \psi_{i}^{\prime \prime} \cos \theta \mathrm{d} \lambda\right)\right) \mathrm{d} x+\int_{0}^{L} \psi_{i}^{\prime} \psi_{j}^{\prime}\left(I_{2} \cos ^{2} \theta+I_{1} \sin ^{2} \theta\right) \mathrm{d} x
\end{aligned}
$$




$$
\begin{aligned}
M_{i, j}^{v, w} & =M_{j, i}^{w, v}=\int_{0}^{L} m\left(\int_{0}^{x} e_{A} \cos \theta \psi_{i}^{\prime \prime} \mathrm{d} \lambda \int_{0}^{x} e_{A} \sin \theta \psi_{j}^{\prime \prime} \mathrm{d} \lambda-\psi_{i}^{\prime} e_{M} \cos \theta \int_{0}^{x} e_{A} \sin \theta \psi_{j}^{\prime \prime} \mathrm{d} \lambda-\right. \\
& \left.\psi_{j}^{\prime} e_{M} \sin \theta \int_{0}^{x} e_{A} \cos \theta \psi_{i}^{\prime \prime} \mathrm{d} \lambda\right) \mathrm{d} x+\frac{1}{2} \int_{0}^{L} \psi_{i}^{\prime} \psi_{j}^{\prime} \sin 2 \theta\left(I_{2}-I_{1}\right) \mathrm{d} x \\
M_{i, j}^{w, w} & =\int_{0}^{L} m\left(\psi_{i} \psi_{j}+\int_{0}^{x} e_{A} \psi_{i}^{\prime \prime} \sin \theta \mathrm{d} \lambda \int_{0}^{x} e_{A} \psi_{j}^{\prime \prime} \sin \theta \mathrm{d} \lambda-e_{M} \sin \theta\left(\psi_{i}^{\prime} \int_{0}^{x} e_{A} \psi_{j}^{\prime \prime} \sin \theta \mathrm{d} \lambda+\right.\right. \\
& \left.\left.\psi_{j}^{\prime} \int_{0}^{x} e_{A} \psi_{i}^{\prime \prime} \sin \theta \mathrm{d} \lambda\right)\right) \mathrm{d} x+\int_{0}^{L} \psi_{i}^{\prime} \psi_{j}^{\prime}\left(I_{2} \sin ^{2} \theta+I_{1} \cos ^{2} \theta\right) \mathrm{d} x
\end{aligned}
$$

The damping matrix $[C]$ is proportional to the rotational speed of the rotor $\Omega$. It is anti-symmetric and related to the effect of Coriolis acceleration. The latter generates accelerations normal to the rotation axis and the velocity of the body. Thus, radial dislocations in the flap direction produces accelerations in the lead-lag direction and vice versa. Due to the anti-symmetry of the matrix, it does not introduce real structural or material damping and effectively acts as a rotating matrix. Therefore, the term gyroscopic will be used instead of damping within the following sections. The individual components of the $[C]$ matrix are defined as follows:

$$
\begin{aligned}
C_{i, j}^{\phi, \phi} & =2 \Omega \int_{0}^{L} m e_{M} \sin \theta\left(\psi_{i} \int_{0}^{x} \frac{J}{A} \theta^{\prime} \psi_{j}^{\prime} \mathrm{d} \lambda-\psi_{j} \int_{0}^{x} \frac{J}{A} \theta^{\prime} \psi_{i}^{\prime} \mathrm{d} \lambda\right) \mathrm{d} x \\
C_{i, j}^{\phi, v} & =-C_{j, i}^{v, \phi}=2 \Omega \int_{0}^{L} m\left(\psi_{i} e_{M}\left(\psi_{j} \beta \cos \theta-\sin \theta \int_{0}^{x} e_{A} \psi_{j}^{\prime \prime} \cos \theta \mathrm{d} \lambda\right)+\psi_{j} \int_{0}^{x} \frac{J}{A} \theta^{\prime} \psi_{i}^{\prime} \mathrm{d} \lambda\right) \mathrm{d} x+ \\
& \Omega \int_{0}^{L} \psi_{i} \psi_{j}^{\prime} \sin 2 \theta\left(I_{2}-I_{1}\right) \mathrm{d} x \\
C_{i, j}^{\phi, w} & =-C_{j, i}^{w, \phi}=2 \Omega \int_{0}^{L} m \psi_{i} e_{M} \sin \theta\left(\beta \psi_{j}-\int_{0}^{x} e_{A} \psi_{j}^{\prime \prime} \sin \theta \mathrm{d} \lambda\right) \mathrm{d} x+ \\
& 2 \Omega \int_{0}^{L} \psi_{i} \psi_{j}^{\prime}\left(I_{2} \sin ^{2} \theta+I_{1} \cos ^{2} \theta\right) \mathrm{d} x \\
C_{i, j}^{v, v} & =2 \Omega \int_{0}^{L} m\left(\left(\psi_{i}^{\prime} \psi_{j}-\psi_{i} \psi_{j}^{\prime}\right) e_{M} \cos \theta+\psi_{i} \int_{0}^{x} e_{A} \psi_{j}^{\prime \prime} \cos \theta \mathrm{d} \lambda-\psi_{j} \int_{0}^{x} e_{A} \psi_{i}^{\prime \prime} \cos \theta \mathrm{d} \lambda\right) \mathrm{d} x \\
C_{i, j}^{v, w} & =-C_{j, i}^{w, v}=-2 \Omega \int_{0}^{L} m \psi_{i}\left(\psi_{j} \beta+\psi_{j}^{\prime} e_{M} \sin \theta-\int_{0}^{x} e_{A} \psi_{j}^{\prime \prime} \sin \theta \mathrm{d} \lambda\right) \mathrm{d} x \\
C_{i, j}^{w, w} & =0
\end{aligned}
$$

The centrifugal stiffening matrix $[F]$, which is proportional to $\Omega^{2}$, is symmetric and positive definite. It quantifies the stiffening of the blade due to the centrifugal force acting on it. The individual components of the $[F]$ matrix are defined as follows:

$$
\begin{aligned}
F_{i, j}^{\phi, \phi} & =-\Omega^{2} \int_{0}^{L} m\left(\int_{0}^{x} \frac{J}{A} \theta^{\prime} \psi_{i}^{\prime} \mathrm{d} \lambda \int_{0}^{x} \frac{J}{A} \theta^{\prime} \psi_{j}^{\prime} \mathrm{d} \lambda+e_{M} \beta\left(\psi_{i} \psi_{j} \sin \theta\left(x+x_{T}+e R\right)+\right.\right. \\
& \left.\left.\cos \theta\left(\psi_{i} \int_{0}^{x} \frac{J}{A} \theta^{\prime} \psi_{j}^{\prime} \mathrm{d} \lambda+\psi_{j} \int_{0}^{x} \frac{J}{A} \theta^{\prime} \psi_{i}^{\prime} \mathrm{d} \lambda\right)\right)\right) \mathrm{d} x+\Omega^{2} \int_{0}^{L} \psi_{i} \psi_{j} \cos 2 \theta\left(I_{2}-I_{1}\right) \mathrm{d} x \\
F_{i, j}^{\phi, v}= & -F_{j, i}^{v, \phi}=-\Omega^{2} \int_{0}^{L} m\left(\psi_{i} e_{M} \sin \theta\left(\psi_{j}^{\prime}\left(x+x_{T}+e R\right)-\psi_{j}\right)+\psi_{j}^{\prime} e_{M} \cos \theta \int_{0}^{x} \frac{J}{A} \theta^{\prime} \psi_{i}^{\prime} \mathrm{d} \lambda-\right. \\
& \int_{0}^{x} e_{A} \psi_{i} \psi_{j}^{\prime \prime} \sin \theta \mathrm{d} \lambda\left(x+x_{T}+e R-\beta e_{M} \sin \theta\right)-\int_{0}^{x} e_{A} \psi_{j}^{\prime \prime} \cos \theta \mathrm{d} \lambda\left(\int_{0}^{x} \frac{J}{A} \theta^{\prime} \psi_{i}^{\prime} \mathrm{d} \lambda+\right. \\
& \left.\left.\psi_{i} e_{M} \beta \cos \theta\right)\right) \mathrm{d} x-\Omega^{2} \int_{0}^{L} \psi_{i} \psi_{j}^{\prime} \beta \cos 2 \theta\left(I_{2}-I_{1}\right) \mathrm{d} x
\end{aligned}
$$




$$
\begin{aligned}
& F_{i, j}^{\phi, w}=-F_{j, i}^{w, \phi}=-\Omega^{2} \int_{0}^{L} m\left(\psi_{i} e_{M} \cos \theta\left(\psi_{j} \beta^{2}-\psi_{j}^{\prime}\left(x+x_{T}+e R\right)-\beta \int_{0}^{x} e_{A} \psi_{j}^{\prime \prime} \sin \theta \mathrm{d} \lambda\right)+\right. \\
&\left(x+x_{T}+e R-e_{M} \beta \sin \theta\right) m \int_{0}^{x} e_{A} \psi_{i} \psi_{j}^{\prime \prime} \cos \theta \mathrm{d} \lambda+m \int_{0}^{x} \frac{J}{A} \theta^{\prime} \psi_{i}^{\prime} \mathrm{d} \lambda\left(\psi_{j} \beta+\right. \\
&\left.\left.\psi_{j}^{\prime} e_{M} m \sin \theta-\int_{0}^{x} e_{A} \psi_{j}^{\prime \prime} \sin \theta \mathrm{d} \lambda\right)\right) \mathrm{d} x-\Omega^{2} \int_{0}^{L} \psi_{i} \psi_{j}^{\prime} \beta \sin 2 \theta\left(I_{2}-I_{1}\right) \mathrm{d} x \\
& F_{i, j}^{v, v}=-\Omega^{2} \int_{0}^{L} m\left(\psi_{i} \psi_{j}-\left(x+x_{T}+e R-\beta e_{M} \sin \theta\right) \int_{0}^{x} \psi_{i}^{\prime} \psi_{j}^{\prime} \mathrm{d} \lambda+\right. \\
& \int_{0}^{x} e_{A} \psi_{i}^{\prime \prime} \cos \theta \mathrm{d} \lambda \int_{0}^{x} e_{A} \psi_{j}^{\prime \prime} \cos \theta \mathrm{d} \lambda-e_{M} \cos \theta\left(\psi_{i}^{\prime} \int_{0}^{x} e_{A} \psi_{j}^{\prime \prime} \cos \theta \mathrm{d} \lambda+\right. \\
&\left.\left.\psi_{j}^{\prime} \int_{0}^{x} e_{A} \psi_{i}^{\prime \prime} \cos \theta \mathrm{d} \lambda\right)\right) \mathrm{d} x-\Omega^{2} \int_{0}^{L} \psi_{i}^{\prime} \psi_{j}^{\prime}\left(I_{2} \cos ^{2} \theta+I_{1} \sin { }^{2} \theta\right) \mathrm{d} x \\
& F_{i, j}^{v, w}=-F_{j, i}^{w, v}=-\Omega^{2} \int_{0}^{L} m\left(\psi_{i}^{\prime} e_{M} \cos \theta\left(\psi_{j} \beta-\int_{0}^{x} e_{A} \psi_{j}^{\prime \prime} \sin \theta \mathrm{d} \lambda\right)-\int_{0}^{x} e_{A} \psi_{i}^{\prime \prime} \cos \theta \mathrm{d} \lambda\left(\psi_{j} \beta+\right.\right. \\
&\left.\left.\psi_{j}^{\prime} e_{M} \sin \theta-\int_{0}^{x} e_{A} \psi_{j}^{\prime \prime} \sin \theta \mathrm{d} \lambda\right)\right) \mathrm{d} x-\frac{1}{2} \Omega^{2} \int_{0}^{L} \psi_{i}^{\prime} \psi_{j}^{\prime} \sin 2 \theta\left(I_{2}-I_{1}\right) \mathrm{d} x \\
& F_{i, j}^{w, w}=-\Omega^{2} \int_{0}^{L} m\left(-\int_{0}^{x} \psi_{i}^{\prime} \psi_{j}^{\prime} \mathrm{d} \lambda\left(x+x_{T}+e R-\beta e_{M} \sin \theta\right)+e_{M} \beta \sin \theta\left(\psi_{i}^{\prime} \psi_{j}+\psi_{j}^{\prime} \psi_{i}\right)-\right. \\
& \psi_{i} \psi_{j} \beta^{2}-\int_{0}^{x} e_{A} \psi_{j}^{\prime \prime} \sin \theta \mathrm{d} \lambda\left(\psi_{i} \beta+\psi_{i}^{\prime} e_{M} \sin \theta\right)-\int_{0}^{x} e_{A} \psi_{i}^{\prime \prime} \sin \theta \mathrm{d} \lambda\left(\psi_{j} \beta+\psi_{j}^{\prime} e_{M} \sin \theta\right)+ \\
&\left.{ }_{0}^{x} \sin \theta \mathrm{d} \lambda \int_{0}^{x} e_{A} \psi_{j}^{\prime \prime} \sin \theta \mathrm{d} \lambda\right) \mathrm{d} x-\Omega^{2} \int_{0}^{L} \psi_{i}^{\prime} \psi_{j}^{\prime}\left(I_{2} \sin { }^{2} \theta+I_{1} \cos ^{2} \theta\right) \mathrm{d} x
\end{aligned}
$$

The structural stiffening matrix $[G]$ is associated with the elastic potential energy of the blade. It is symmetric and positive definite. The individual components of the $[G]$ matrix are defined as follows:

$$
\begin{aligned}
G_{i, j}^{\phi, \phi} & =\int_{0}^{L}\left(G J+\frac{J}{A} \int_{x}^{L} m \Omega^{2}(\lambda+e R) \mathrm{d} \lambda+E \theta^{\prime 2}\left(B_{1}^{*}-\frac{J^{2}}{A}\right)\right) \psi_{i}^{\prime} \psi_{j}^{\prime} \mathrm{d} x+K^{\phi} \psi_{i_{0}} \psi_{j_{0}} \\
G_{i, j}^{\phi, v} & =G_{j, i}^{v, \phi}=-\int_{0}^{L} E \theta^{\prime} \cos \theta\left(B_{2}^{*}-J e_{A}\right) \psi_{i}^{\prime} \psi_{j}^{\prime \prime} \mathrm{d} x \\
G_{i, j}^{\phi, w} & =G_{j, i}^{w, \phi}=-\int_{0}^{L} E \theta^{\prime} \sin \theta\left(B_{2}^{*}-J e_{A}\right) \psi_{i}^{\prime} \psi_{j}^{\prime \prime} \mathrm{d} x \\
G_{i, j}^{v, v} & =\int_{0}^{L} E\left(I_{y} \sin ^{2} \theta+\left(I_{z}-A e_{A}^{2}\right) \cos ^{2} \theta\right) \psi_{i}^{\prime \prime} \psi_{j}^{\prime \prime} \mathrm{d} x+K^{v} \psi_{i_{0}}^{\prime} \psi_{j_{0}}^{\prime} \\
G_{i, j}^{v, w} & =G_{j, i}^{w, v}=\frac{1}{2} \int_{0}^{L} E \sin 2 \theta\left(\left(I_{z}-A e_{A}^{2}\right)-I_{y}\right) \psi_{i}^{\prime \prime} \psi_{j}^{\prime \prime} \mathrm{d} x \\
G_{i, j}^{w, w} & =\int_{0}^{L} E\left(I_{y} \cos ^{2} \theta+\left(I_{z}-A e_{A}^{2}\right) \sin ^{2} \theta\right) \psi_{i}^{\prime \prime} \psi_{j}^{\prime \prime} \mathrm{d} x+K^{w} \psi_{i_{0}}^{\prime} \psi_{j_{0}}^{\prime}
\end{aligned}
$$

Finally, an external static forcing vector $\{f\}$ is obtained through the application of Lagrange's equation of motion to the kinetic energy of the system. This time-invariant vector represents the static deflection associated with by the centrifugal force. The deviation of the mass centre of the blade from the radially outward location where the centrifugal load is applied generates this static deflection. Therefore, coupling parameters such as pretwist, precone, and offset between mass centre and elastic axis are effectively associated with the static vector $\{f\}$. It is noted that this vector does not influence the natural vibration characteristics of the rotor blade due to the steady nature of the forcing. The individual components of the $[f]$ vector are defined as follows: 


$$
\begin{aligned}
f_{i}^{\phi}= & -\Omega^{2} \int_{0}^{L} m\left(\left(x+x_{T}+e R-\beta e_{M} \sin \theta\right) \int_{0}^{x} \frac{J}{A} \theta^{\prime} \psi_{i}^{\prime} \mathrm{d} \lambda+\left(x+x_{T}+e R\right) \psi_{i} e_{M} \beta \cos \theta\right) \mathrm{d} x- \\
& \frac{1}{2} \Omega^{2} \int_{0}^{L} \psi_{i} \sin 2 \theta\left(I_{2}-I_{1}\right) \mathrm{d} x \\
f_{i}^{v} & =\Omega^{2} \int_{0}^{L} m\left(e_{M} \cos \theta\left(\psi_{i}-\psi_{i}^{\prime}\left(x+x_{T}+e R\right)\right)+\right. \\
& \left.\int_{0}^{x} e_{A} \psi_{i}^{\prime \prime} \cos \theta \mathrm{d} \lambda\left(x+x_{T}+e R-\beta e_{M} \sin \theta\right)\right) \mathrm{d} x+\frac{1}{2} \Omega^{2} \int_{0}^{L} \psi_{i}^{\prime} \beta \sin 2 \theta\left(I_{2}-I_{1}\right) \mathrm{d} x \\
f_{i}^{w}= & -\Omega^{2} \int_{0}^{L} m\left(\left(x+x_{T}+e R-\beta e_{M} \sin \theta\right)\left(\psi_{i} \beta-\int_{0}^{x} e_{A} \psi_{i}^{\prime \prime} \sin \theta \mathrm{d} \lambda\right)+\right. \\
& \left.\left(x+x_{T}+e R\right) \psi_{i}^{\prime} e_{M} \sin \theta\right) \mathrm{d} x+\Omega^{2} \int_{0}^{L} \psi_{i}^{\prime} \beta\left(I_{2} \sin ^{2} \theta+I_{1} \cos ^{2} \theta\right) \mathrm{d} x
\end{aligned}
$$

To obtain the natural frequencies and mode shapes of vibration, an eigenvalue problem is generated and solved. Inserting a generic solution of the form $\{q\}=\{\bar{q}\} \exp (\lambda t)$ in the homogeneous part of the second order system of linear differential equations defined in Eq. (15) leads to the following quadratic eigenvalue problem (QEP)

$$
\left(\lambda^{2}[M]+\lambda[C]+[S]\right)\{\bar{q}\}=[Q(\lambda)]\{\bar{q}\}=\{0\}
$$

where $\lambda$ represents an eigenvalue and $\{\bar{q}\}$ is its associated eigenvector. The dimension of the $\lambda$-matrix $[Q(\lambda)]$ is $3 N \times 3 N$, where $N$ is the number of assumed deformation functions per DOF. The symmetry of $[M]$ and $[S]$ and skew-symmetry of $[C]$ determine the spectrum location of the $6 N$ eigenvalues in the complex plane. The eigenvalues are purely imaginary $(\Re(\lambda)=0)$ and symmetrically located with respect to the real axis as reported in Ref. 19. Hence, the eigenvalues and eigenvectors occur in pairs of conjugate complex numbers $\lambda, \bar{\lambda}$ and $\{\bar{q}\}, \bar{q}\}$. The final $N$ first mode shapes are calculated as dot products of the assumed deformation function vectors and the eigenvectors of the QEP as shown in Eq. (22).

$$
X_{i}^{r}(x)=\left\{\psi^{r}(x)\right\} \cdot\left\{\bar{q}^{r}(t)\right\}_{i}, \quad i=1, \ldots N
$$

\section{Results and discussion}

\subsection{Numerical performance}

The numerical performance of the proposed method has been assessed by analysing the convergence of the natural frequencies on the number of assumed deformation functions per DOF. A small scale, $1.92 \mathrm{~m}$ diameter, torsionally soft, untwisted hingeless helicopter rotor blade was used as case study. The blade was designed to have uniform properties from $9.5 \%$ of the blade radius to the tip. The two bladed rotor was tested at hover conditions at different rotor speeds ranging from 0 to 1000rpm. The objective of the analysis described in Ref. 20 was to quantify the modal frequencies, steady blade bending moments, and lead-lag damping.

Figure 3 presents the influence of the number of assumed deformation functions per DOF on the convergence natural frequencies. The natural frequencies were normalized with the nominal rotorspeed $\left(\Omega=105 \mathrm{rad} \mathrm{s}^{-1}\right)$. In Fig. 3, the labels refer to the dominant DOF. The first two natural modes (1F and $1 \mathrm{~L}$ ) are highly independent of the number of assumed deformation functions. Convergence of the normalized natural frequencies is attained with only two assumed deformation functions per DOF, and no further variation is obtained as a result of the use of a higher number of assumed deformation functions. The estimation of the first 7 natural modes of vibration( $1 \mathrm{~F}, 1 \mathrm{~L}, 1 \mathrm{~T}, 2 \mathrm{~F}, 3 \mathrm{~F}, 2 \mathrm{~T}$, and $2 \mathrm{~L})$ is attained with the use of 4 modes per DOF. The calculation of high-order content requires the deployment of a larger number of assumed deformation functions, e.g. 7 assumed deformation functions per DOF are required to reach convergence for the 12 first natural modes. 


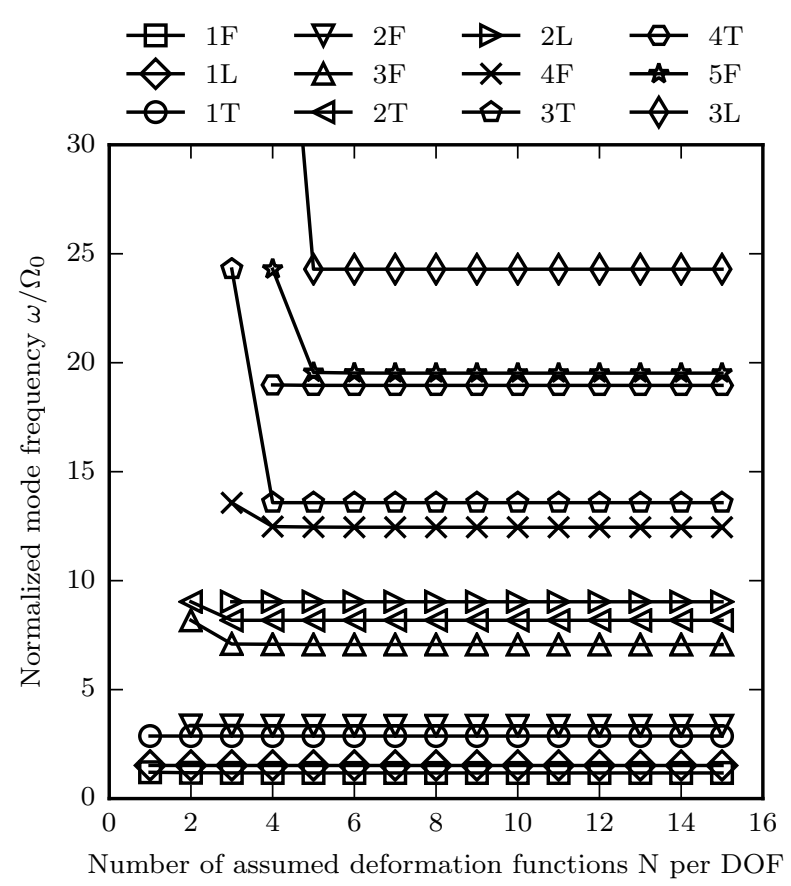

Fig. 3. Influence of number of assumed deformation functions for the hingeless rotor blade model described in Ref. 20 on the convergence of the normalized modal frequencies.

Thus, the proposed method has been proven to exhibit excellent numerical behaviour. A relatively small number of assumed deformation functions per DOF is required for the convergence of the final natural frequencies. This characteristic is highly associated with the deployment of assumed deformation functions derived from classical methods. These better approximate the actual mode shape accelerating the convergence of the method. The analytical availability of the first and second assumed deformation function spatial derivatives does not introduce any numerical error. As a result, an infinite number of vibration modes can be theoretically calculated. Additionally, the lack of numerical instabilities found in the analyses carried out, presents this method as a robust approach for the structural treatment of helicopter rotor blades.

\subsection{Computational performance}

The computational performance of the method has been evaluated by analysing the dependence of the required computational time on the number of assumed deformation functions. The small scale hingeless rotor described in Ref. 20 has been used as study case. The measured computational time comprises the allocation of system variables, solution of the classical methods, population of the dynamic system matrices, solution of the QEP, eigenvector normalization and calculation of the final mode shapes. The method has been implemented in serial FORTRAN 90/95 code. The calculations have been carried out on a personal computer equipped with 8 Gigabytes (GB) of Random Access Memory (RAM) and a Central Processing Unit (CPU) operating at 1.7 Gigahertz $(\mathrm{GHz})$ without parallel processing.

Figure 4 presents the evolution of the required computational time with the number of assumed deformation functions. A $N^{2}$ relation is identified. Thus, the convergence of the method needs to be quick to avoid computational overhead. Figure 3 shows that, the first 12 natural modes can be estimated with the use of 7 assumed modes per DOF. This fairly quick convergence requires a computational time of 0.9 seconds. If a lower number of vibration modes is desired, e.g. up to 7 th mode, the computational time would be reduced to only half a second. 


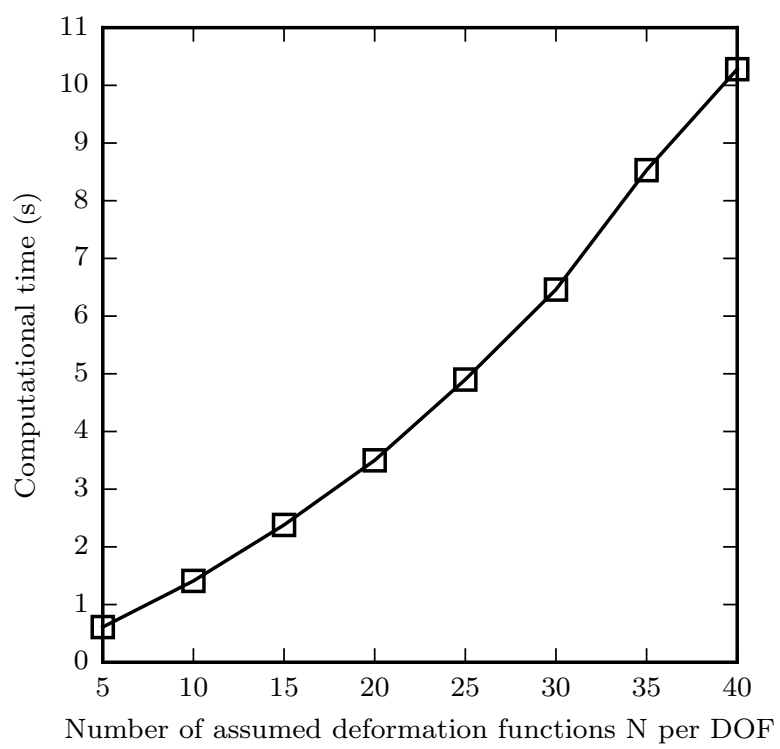

Fig. 4. Influence of number of assumed deformation functions for the hingeless rotor blade model described in Ref. 20 on: the required computational time for a personal computer with $1.7 \mathrm{GHz}$ CPU and $8 \mathrm{~GB}$ of RAM.

Excellent computational performance has been identified for the proposed method. Fully converged solutions with 7 modes of vibration have been obtained in half a second on a low-end personal computer for the configurations studied. The excellent computational performance enables an efficient integration of the method into a rotorcraft comprehensive code.

\subsection{Comparison with experiment and FEA}

The agreement of the proposed method results with experimental measurements and FEA results is assessed for two small scale hingeless rotor blades. These are the previously presented torsionally soft, untwisted blade described in Ref. 20 and the HART II rotor blade. The latter is a $2 \mathrm{~m}$ radius, geometrically and dynamically scaled model of the hingeless BO-105 helicopter rotor blade used in the Higher harmonic control Aeroacoustics Rotor Test program HART II. The main objective of the HART II program was to generate a comprehensive database of rotor loads, pressure distributions, blade deformations, acoustic radiations, and wind tunnel data for the small scale rotor during descending flight. A detailed description of the HART II rotor blade can be found in Ref. 21.

Figure 5 presents the resonance chart calculated for the small scale, torsionally soft, hingeless rotor described in Ref. 20. Natural frequencies and rotorspeed have been normalized with its nominal rotorspeed $\Omega_{0}=105 \mathrm{rad} \mathrm{s}^{-1}$. Solid and dashed lines represent the natural frequencies predicted with the present approach and FEA respectively. Experimental measurements in vacuum reported in Ref. 22 have been labelled as Exp. Excellent agreement between experimental measurement and results calculated by the proposed method is observed. The averaged relative errors between both methods across the whole rotorspeed range are of the order of $0.3 \%, 1.6 \%, 0.5 \%, 1.7 \%$, and $0.5 \%$ for the first 5 vibration modes respectively. The comparison of calculations of the proposed method with FEA results reported in Ref. 22 leads to the identification of average relative errors of the order of $1.1 \%, 1.0 \%, 1.3 \%, 0.5 \%$, and $0.5 \%$. It is noted that, the order of magnitude of the relative errors is representative of a method with excellent agreement with FEA.

The proposed method is able to predict transitions between modes of vibration, i.e. changes in the dominant DOF for a given natural mode of vibration. The transition between $2 \mathrm{~F}$ and $1 \mathrm{~T}$ modes can be observed in the magnified area of Fig. 5 as the convergence and posterior divergence of the frequencies of both modes. Apart from the convergence-divergence of the natural frequencies, an energy transfer takes place. The flapping amplitude is reduced whilst the torsional deformation is 


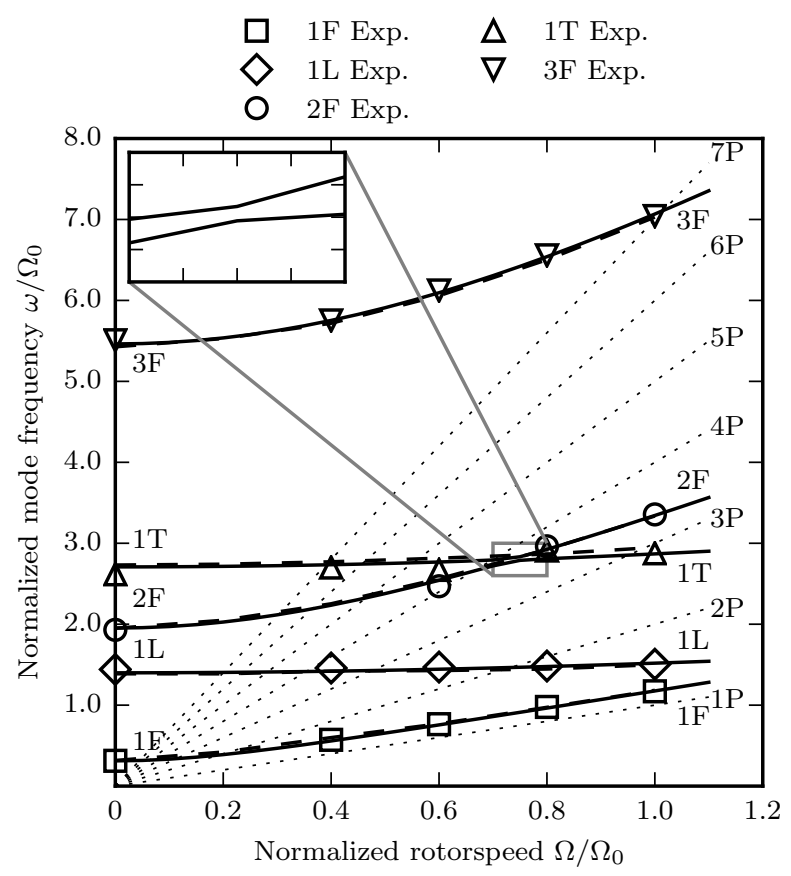

Fig. 5. Calculated resonance chart for the hingeless rotor blade model described in Ref. 20. - Comparison with experimental measurements and FEA results from Ref. 22 - Solid and dashed lines denote Lagrangian and FEA predictions respectively.

increased for the 3rd natural mode in the convergent region. This process continues in the divergent region until the torsion becomes the dominant DOF. The opposite phenomenon takes place for the 4 th natural mode. This can only be predicted with methods able to model coupling between modes of vibration such as the proposed approach.

Figure 6 presents the calculated dominant mode shapes for the first 5 modes of vibration of the hingeless rotor described in Ref. 20 at nominal speed. Very good agreement with experimental and FEA data reported in Ref. 22 is observed for all modes except for the 1st flap mode where a significant variation in mode shape is noticed.

Figure 7 presents the resonant chart calculated for small scale hingeless HART II rotor blade. Natural frequencies and rotorspeed have been normalized with the nominal rotorspeed $\Omega_{0}=109.01 \mathrm{rad} \mathrm{s}^{-1}$. Solid lines represent natural frequencies calculated with the present approach. Experimental measurements taken in vacuum for the 2 nd to 6 th vibration modes (Refs. 23 and 24) have been superimposed in Fig. 7 as Exp. Experimental tests have been only undertaken at non-rotating conditions and therefore no comparison can be done at higher rotorspeeds. It is noted that the experimental measurements of the 1 st torsion mode present scatter. This has been attributed to variations in blade weight consequence of the attachment of instrumentation to the blade (Ref. 21). The calculated natural frequencies exhibit excellent agreement with the experimental measurements. Relative errors of the order of $0.4 \%, 0.5 \%, 1.1 \%, 4 \%, 1.4 \%$, and $1.7 \%$ are observed for the 2 nd to 6 th natural modes respectively. For this particular configurations three different transitions between modes of vibration are observed. The 1 st one involves $1 \mathrm{~F}$ and $1 \mathrm{~L}$ and is magnified at the upper left hand corner). The 2 nd one involves $1 \mathrm{~T}$ and $3 \mathrm{~F}$ and is magnified at the top of the figure. Finally a 3rd transition between $2 \mathrm{~L}$ and $3 \mathrm{~F}$ is identified.

Potential sources of errors between the estimated frequencies and the experimental measurements and FEA results can be identified. The first one is the truncation scheme deployed during the derivation of the presented formulation, terms involving transverse displacement and/or torsion deformation of order cubic or higher have been eliminate. That leads to the omission of certain nonlinear terms which might be relevant at high speed conditions. Shear deformation, rotary inertia, and other secondary nature nonlinear terms have also been neglected. Those could lead to significant variation in predicted natural frequencies at high speed. A third source of error is the normalization condition applied to the assumed 


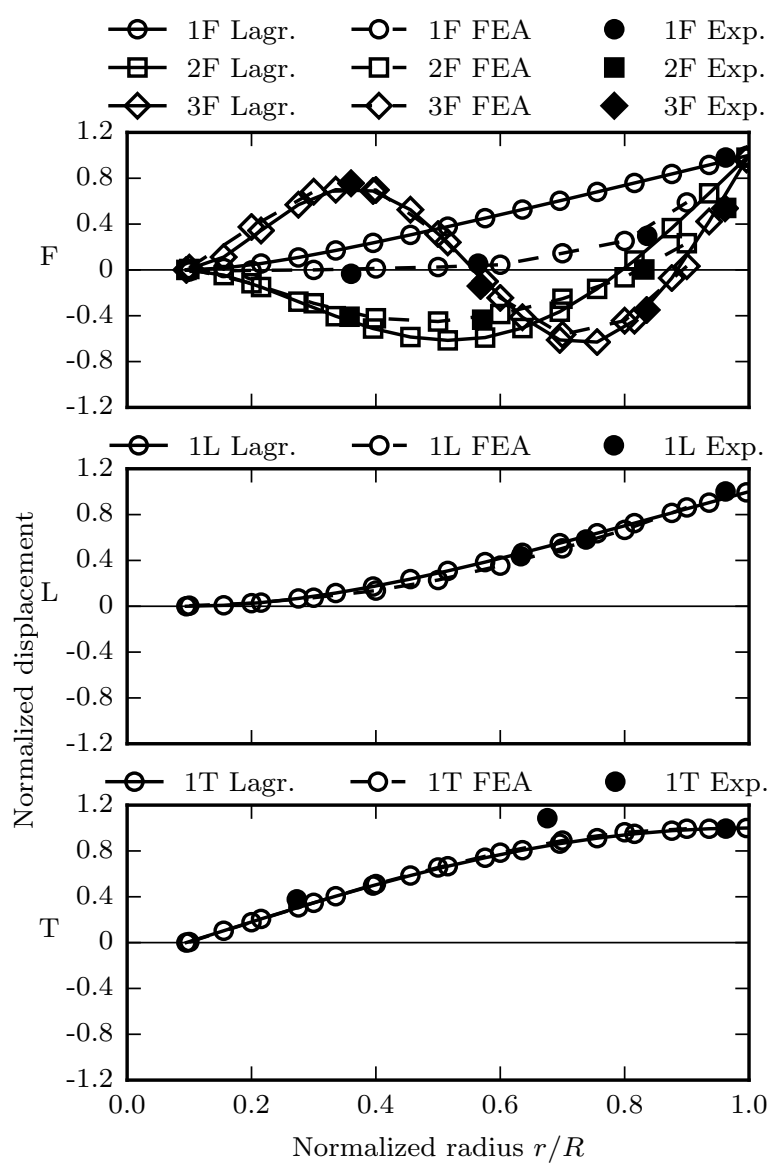

Fig. 6. Normalized dominant mode shapes for the hingeless rotor blade model described in Ref. $20, \Omega=105 \mathrm{rad} \mathrm{s}^{-1}$ - Comparison with experimental measurements and FEA results from Ref. 22.

deformation functions, for small scale rotors the assumption of generalized mass equal to 1.0 can lead to a potential breakdown of the linearisation associated with the small displacement formulation as reported in Ref. 15. A fourth source of error is the uncertainty of some inertial and structural properties not readily available in the public domain, which have been estimated based on sensible best guess engineering criteria. These include: blade cross-sectional integrals $B_{1,2}$, area centroid offset from the elastic axis $e_{A}$, extensional stiffness $E A$, and blade mass moments $I_{1,2}$. A fifth source of error is the uncertainty of the experimental measurements itself. The introduction of instrumentation can cause variations in the vibration characteristics of the rotor blade. Additionally, a deficient vacuum isolation can lead to deviation in the modal characteristics due to aerodynamic damping.

\subsection{Comparison with multi-body analysis methods}

The proposed approach is compared with multi-body analysis methods extensively used in comprehensive rotorcraft codes. To carry out this comparison, a resonant chart is calculated for the small scale hingeless HART II rotor described in Ref. 21. Additionally, the dominant mode shapes are calculated and compared at its nominal rotational speed.

Figure 7 presents the resonant chart for the HART II rotor. Natural frequencies and rotational speed have been normalized with the nominal speed $\Omega_{0}=109.01 \mathrm{rad} \mathrm{s}^{-1}$. Solid and dashed lines represent predictions of the proposed method and CAMRAD calculations respectively. The averaged relative errors across the rotorspeed range are of the order of 3\%, $2 \%$, $3 \%, 2 \%, 4 \%, 4 \%$, and $5 \%$ for the first 7 natural frequencies. The larger deviations from CAMRAD calculations can be 


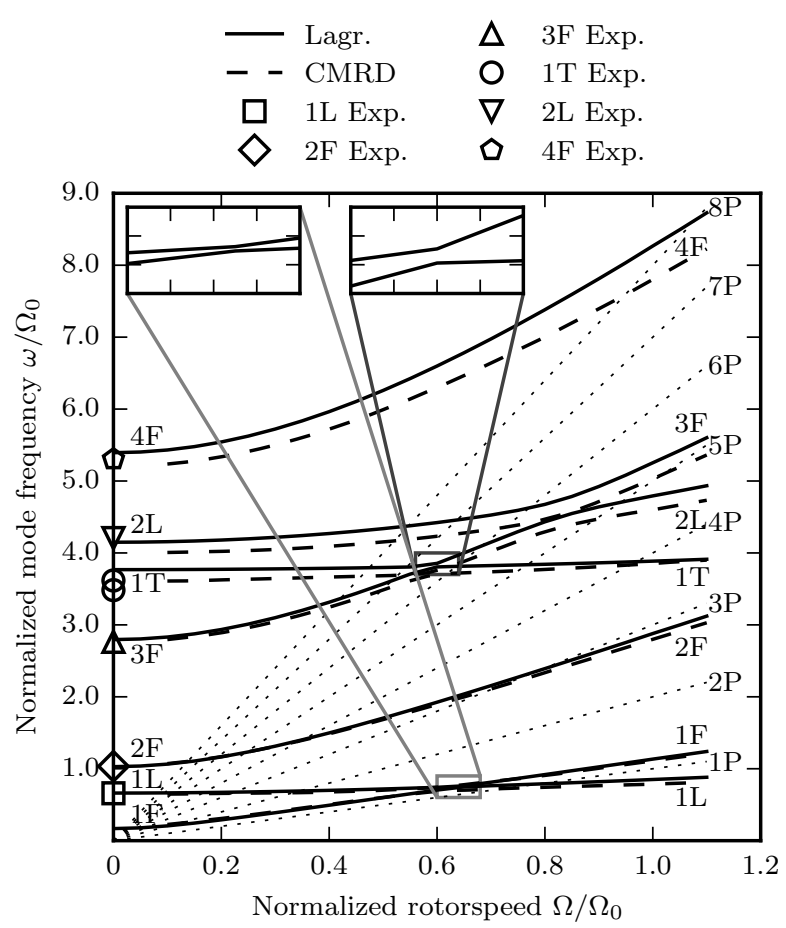

Fig. 7. Calculated resonance charts for the HART II rotor described in Ref. 21 - comparison with CAMRAD calculation from Ref. 25 and experimental measurements from Refs. 23, 24 - Solid and dashed lines denote Lagrangian and CAMRAD predictions respectively.

justified by the deployment of different sets of mass and structural properties. The calculations carried out with CAMRAD, reported in Ref. 25, were based on the original set of structural data (Ref. 24). Jung et al. (Ref. 21) further studied the structural properties and found that the initial documentation overestimated the properties by 5 to $15 \%$. The structural properties obtained by Jung et al. (Ref. 21) have been used in the present work. The excellent agreement of the Lagrangian estimation with the experimental measurements shown in Fig. 7 justifies the use of the newest set of structural properties.

Figure 8 shows the calculated dominant mode shapes for the first 5 modes of vibration of the small hingeless HART II rotor blade at its nominal speed. Excellent agreement in the shape and amplitude of the modes estimated by both means of the proposed approach and multi-body analysis methods is observed for all the natural modes.

The natural frequencies estimated with proposed approach exhibit good agreement with those calculated with multibody dynamics codes. Discrepancies of the order of 3\%, justified by the use of different structural properties, have been identified for the natural frequencies of this small scale rotor. Excellent agreement between mode shapes calculated with both methods is observed. The overall good agreement of both techniques makes the proposed approach a computationally efficient alternative to multi-body dynamics.

\subsection{Coupling analysis}

As a consequence of the symmetry of the mass and stiffness matrix and the antisymmetry or the gyroscopic damping matrix, pairs of complex conjugate eigenvalues and eigenvectors are obtained solving the QEP of Eq. (21). If the gyroscopic matrix is eliminated, denominated as the undamped case, and a solution of the form $\{q\}=\{\bar{q}\} \sin \lambda t$ is assumed, the QEP is reduced to the following generalized eigenvalue problem (GEP)

$$
\left([S]-\lambda^{2}[M]\right)\{\bar{q}\}=[Q(\lambda)]\{\bar{q}\}=\{0\}
$$




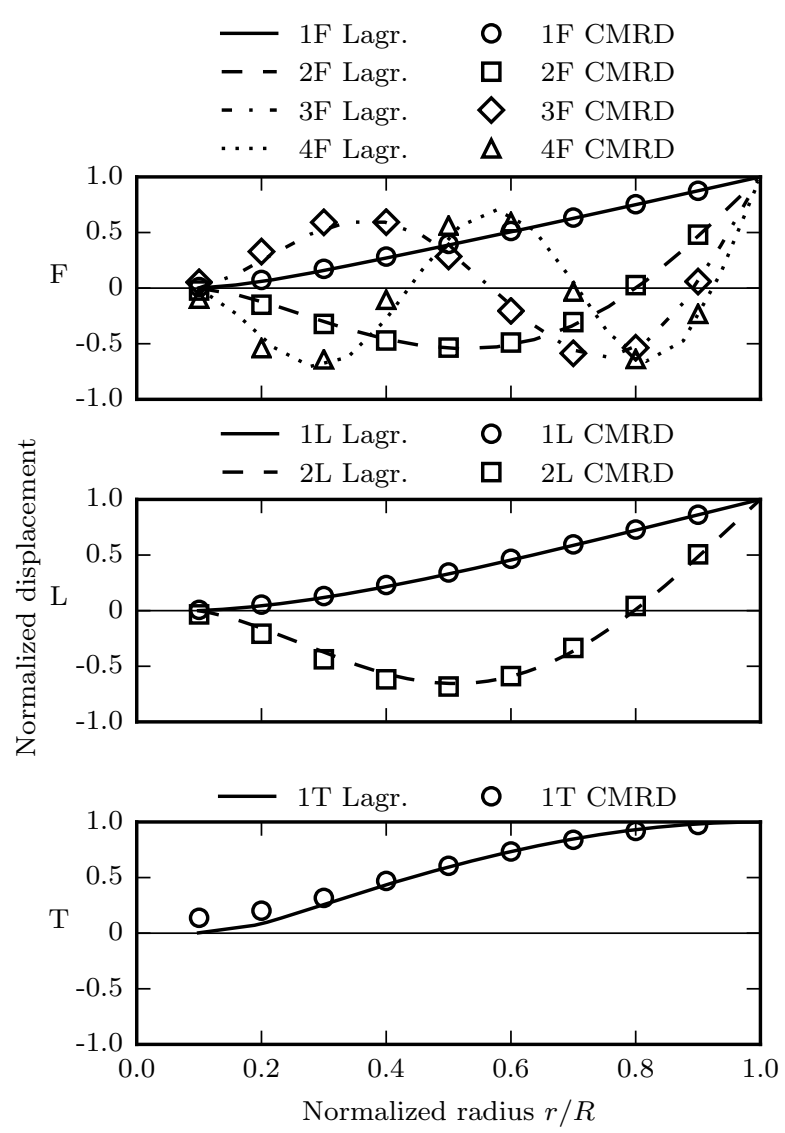

Fig. 8. Normalized dominant mode shapes for the hingeless rotor blade model described in Ref. $21, \Omega=109.01 \mathrm{rad} \mathrm{s}^{-1}-\mathrm{comparison}^{-1}$ with CAMRAD calculations from Ref. 25.

where $\lambda$ is a real eigenvalue and $\{\bar{q}\}$ its associated real right eigenvector. Equation (24) presents the resultant undamped mode equation as meant to be introduced in the forced response of the dynamic system, where $\lambda_{i}$ and $X_{i}^{r}(x)$ are real numbers representing the eigenvalues and final mode shapes calculated with Eq. (22).

$$
\chi_{i}^{r, U}(x, t)=X_{i}^{r}(x) \cos \left(\lambda_{i} t-\varphi_{i}^{r e f}\right)
$$

In Eq. (24), $\chi_{i}^{r, U}(x, t)$ is the undamped $(U)$ time-dependent transverse displacement/torsional deformation for the $i$ th natural mode and $r$ DOF. A reference phase angle $\varphi_{i}^{\text {ref }}$ accounts for the possible phase angle respect the exciting force. This phase angle is unique for all DOFs for a given natural mode and does not depend on the axial position.

If the gyroscopic matrix is included in the analysis, pairs of complex conjugate eigenvalues $\left(\lambda_{i}, \overline{\lambda_{i}}\right)$ and mode shapes $\left(X_{i}^{r}(x), \overline{X_{i}^{r}}\right)$ are obtained. The superimposition of pairs of complex conjugate time-dependent functions $\left(X_{i}^{r}(x) \exp \left(\lambda_{i} t\right), \overline{X_{i}^{r}}(x) \exp \left(\overline{\lambda_{i}} t\right)\right)$ leads to Eq. (25). Where $\chi_{i}^{r, D}(x, t)$ represents the damped $(D)$ time-dependent transverse displacement/torsional deformation for the $i$ th natural mode and $r$ DOF.

$$
\chi_{i}^{r, D}(x, t)=\left|X_{i}^{r}(x)\right| \cos \left(\Im\left(\lambda_{i}\right) t-\varphi_{i}^{r e f}+\arctan \frac{\Im\left(X_{i}^{r}(x)\right)}{\Re\left(X_{i}^{r}(x)\right)}\right) e^{\Re\left(\lambda_{i}\right) t}
$$

As previously discussed in subsection "Eigenproblem", a QEP with symmetric mass and stiffness matrices, and skewsymmetric gyroscopic matrix is associated with pairs of purely imaginary $\left(\Re\left(\lambda_{i}\right)=0\right)$ eigenvalues. As a result, the time-dependent decay term $\exp \left(\Re\left(\lambda_{i}\right) t\right)=1$ and no damping is present. Although the gyroscopic matrix does not introduce any time-dependent decay, it introduces a phase angle $\varphi_{i}^{r}(x)=\arctan \frac{\Im\left(X_{i}^{r}(x)\right)}{\Re\left(X_{i}^{r}(x)\right)}$ which depends exclusively 
Table 1: Influence of gyroscopic damping on the normalized modal frequencies of the model described in Ref. 20.

\begin{tabular}{lllllllllll}
\hline & \multicolumn{2}{c}{ Uncoupled } & \multicolumn{2}{c}{$e_{M}=5 \% c$} & \multicolumn{2}{c}{$\theta_{0}=5 \mathrm{deg}$} & \multicolumn{2}{c}{$\theta^{\prime}=-5 \mathrm{deg}$} & \multicolumn{2}{c}{$\beta=5 \mathrm{deg}$} \\
\cline { 2 - 10 } & $\mathrm{Q}\left(\frac{\omega}{\Omega_{0}}\right)$ & $\mathrm{G}(\%)$ & $\mathrm{Q}\left(\frac{\omega}{\Omega_{0}}\right)$ & $\mathrm{G}(\%)$ & $\mathrm{Q}\left(\frac{\omega}{\Omega_{0}}\right)$ & $\mathrm{G}(\%)$ & $\mathrm{Q}\left(\frac{\omega}{\Omega_{0}}\right)$ & $\mathrm{G}(\%)$ & $\mathrm{Q}\left(\frac{\omega}{\Omega_{0}}\right)$ & $\mathrm{G}(\%)$ \\
\cline { 2 - 10 } $1 \mathrm{~F}$ & 1.176 & 0.000 & 1.176 & 0.000 & 1.170 & 0.000 & 1.174 & 0.000 & 1.173 & 1.555 \\
$1 \mathrm{~L}$ & 1.516 & 0.000 & 1.516 & 0.002 & 1.521 & 0.001 & 1.517 & 0.000 & 1.540 & -1.530 \\
$1 \mathrm{~T}$ & 2.864 & 0.002 & 2.839 & 0.002 & 2.862 & 0.001 & 2.865 & 0.002 & 2.864 & 0.002 \\
$\mathrm{~F}$ & 3.341 & -0.002 & 3.398 & -0.002 & 3.340 & -0.002 & 3.343 & -0.002 & 3.340 & 0.018 \\
$3 \mathrm{~F}$ & 7.064 & 0.001 & 6.971 & 0.000 & 7.064 & 0.001 & 7.054 & 0.001 & 7.064 & 0.003 \\
\hline
\end{tabular}
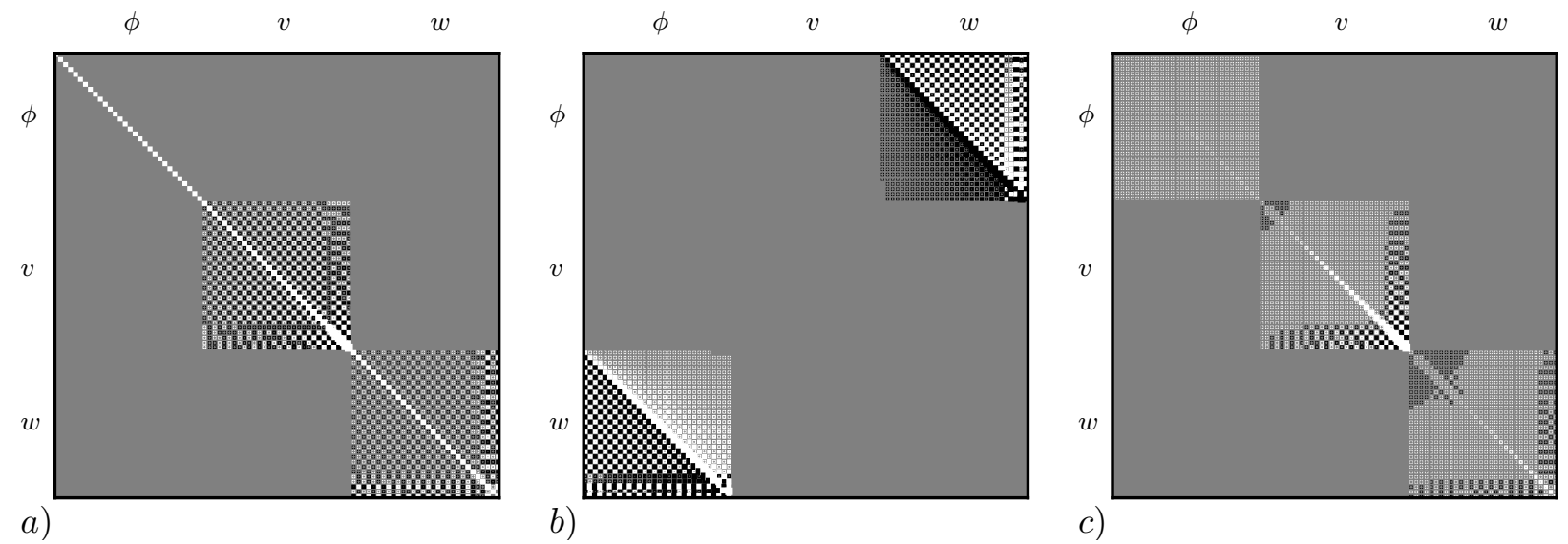

Fig. 9. Hinton diagrams computed for the hingeless rotor blade model described in Ref. 20 - Uncoupled. a) Mass matrix, b) Gyroscopic matrix, c) Overall stiffness matrix.

on the phase angle of the complex number $X_{i}^{r}(x)$. The variation of the phase angle $\varphi_{i}^{r}(x)$ across the blade leads to a time-dependent mode shape variation. Therefore, the mode shape is not constant with a sinusoidal amplitude oscillation as it was observed for the undamped system. In addition to the non-uniformity along the span, the phase angle $\varphi_{i}^{r}(x)$ depends on the DOF $r$ and phase delays between DOFs can be found.

Table 1 quantifies the effect of the inclusion of the gyroscopic matrix on the normalized natural frequencies of the hingeless small scale generic helicopter rotor blade model described in Ref. 20. The analyses were carried out at the nominal speed $\left(\Omega_{0}=105 \mathrm{rad} \mathrm{s}^{-1}\right)$. Normalized frequencies obtained from the QEP are shown in columns labelled as Q $\left(\frac{\omega}{\Omega_{0}}\right)$ whilst the solutions of the GEP are presented as percentage deviations from the QEP data and labelled as G (\%). The uncoupled case refers to a case study with zero offset between the centre of gravity and the elastic axis $\left(e_{M}\right)$, blade pitch angle $\left(\theta_{0}\right)$, pretwist $\left(\theta^{\prime}\right)$. and pre-cone $(\beta)$. Whilst the coupling source of the other cases is specified in the top row.

For the uncoupled case, small discrepancies between the QEP and GEP frequencies are observed for the first torsion (1T) and second flap (2F) modes of vibration. That suggests a soft gyroscopic coupling flapwise displacement and torsional deformation. To corroborate this assumption, Hinton diagrams of the mass, gyroscopic and overall stiffness matrices are presented in Fig. 9 for this case study. A Hinton diagram represents the magnitude and sign of each matrix element with a square. The area of the square is proportional to the absolute value of the matrix element, while the sign of the element is determined by the color (white for positive values and black for negatives). As previously stated, each matrix comprises 9 partitions. These have been arranged as follows: 1st partition column/row corresponds to torsion, 2nd partition column/row to lead-lag, and 3rd partition column/row to flap. From Fig. 9 it is easy to observe the symmetry of the mass and overall stiffness matrices and the anti-symmetry of the gyroscopic matrix. Thus, $M_{i, j}^{r, s}=M_{j, i}^{s, r}, S_{i, j}^{r, s}=S_{j, i}^{s, r}$, and $C_{i, j}^{r, s}=-C_{j, i}^{s, r}$. In Fig. 9 only the diagonal blocks are non-zero for the mass and stiffness matrices. However, a non-diagonal block appears 

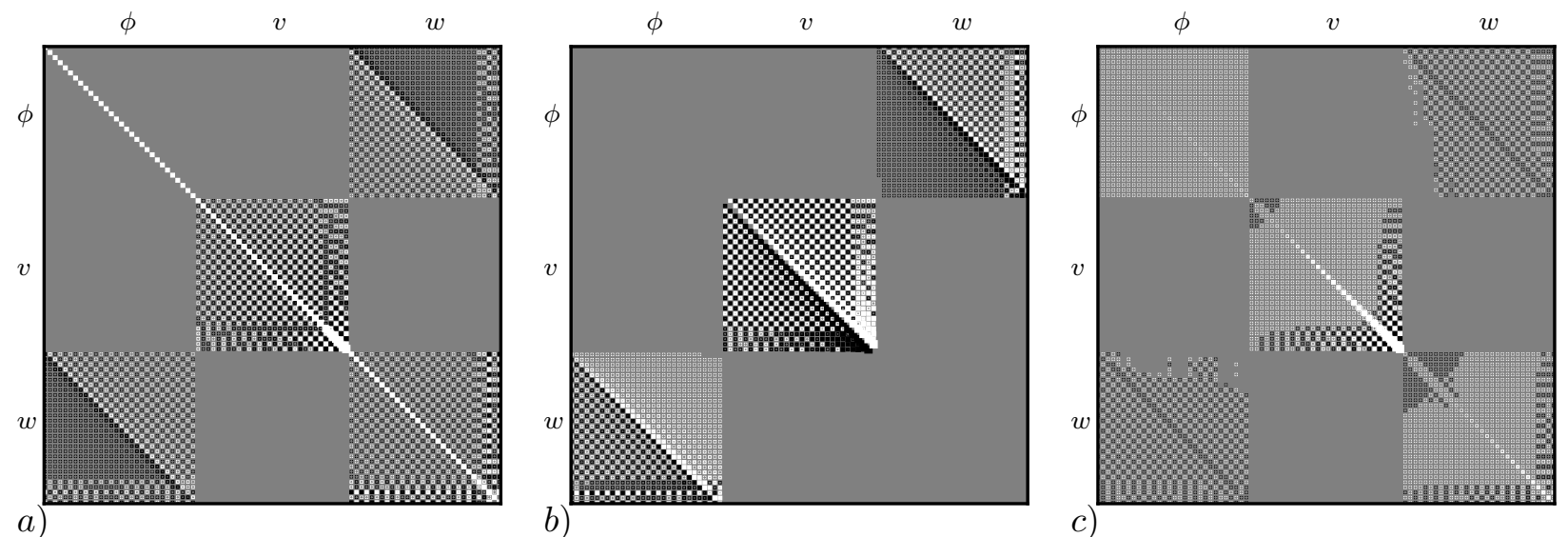

Fig. 10. Hinton diagrams computed for the hingeless rotor blade model described in Ref. $20-e_{M}=5 \% c$. a) Mass matrix, b) Gyroscopic matrix, c) Overall stiffness matrix.

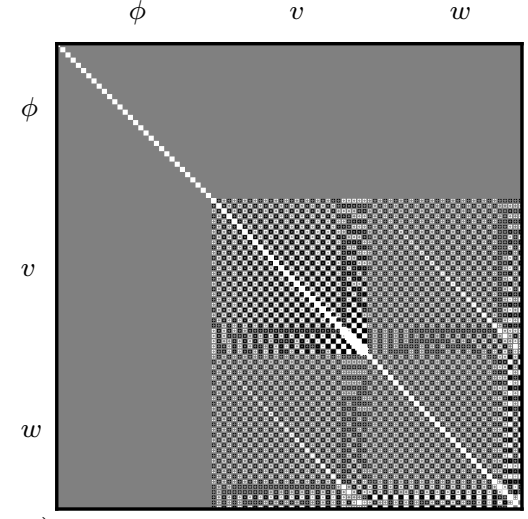

a)

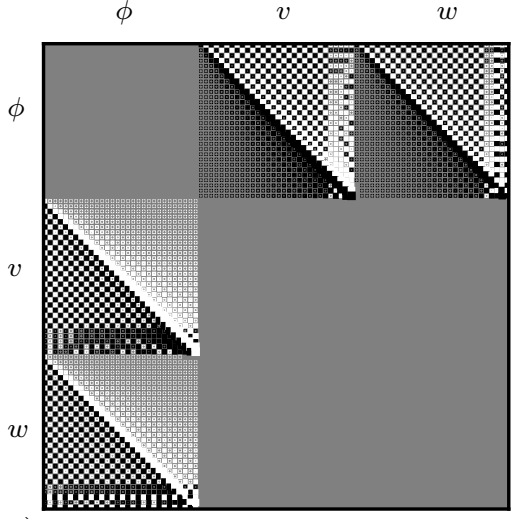

b)

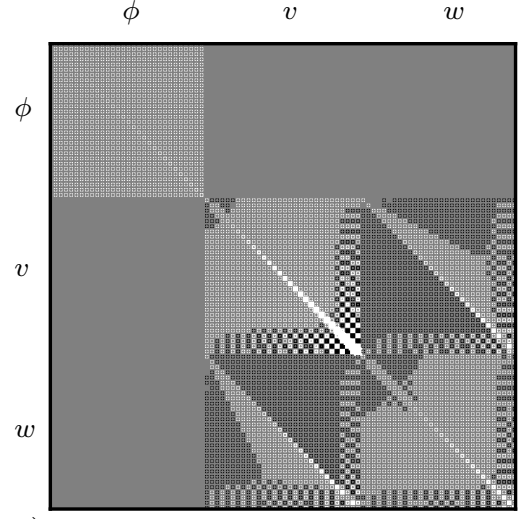

c)

Fig. 11. Hinton diagrams computed for the hingeless rotor blade model described in Ref. 20 - $\theta_{0}=5$ deg. a) Mass matrix, b) Gyroscopic matrix, c) Overall stiffness matrix.

in the gyroscopic matrix which couples flap and torsion. The coupling strength increases with the proximity of the flap and torsion modes in the frequency spectrum and therefore the intensity of the coupling is higher for the coupling between 1T and $2 \mathrm{~F}$ for this particular case of study.

As a result of the inclusion of an offset between the center of gravity and the elastic axis of 5\%, certain coupling between flap and torsion is generated. It can be observed in Table 1, where the first torsion and second flapping frequencies are affected. It can be observed as well in Fig. 10, where blocks coupling flap and torsion appear in the mass and stiffness matrices. Although of low order of magnitude, intermodal coupling between lead-lag assumed deformation functions are observed in the gyroscopic matrix.

The inclusion of blade pitching angle results in coupling between flapwise and chordwise displacements. It can be easily observed in the mass and stiffness matrices of Fig. 11, where blocks linking flap and lag are present. This leads to discrepancies between QEP and GEP frequencies as shown in Table 1. Certain gyroscopic coupling is further generated between lead-lag and torsion. However, the effect is fairly weak due to the distance between torsion and lead-lag frequencies in the frequency spectrum. Although of different nature, the effects of pretwist along the blade and pitching angle are similar and further discussion is not carry out.

The inclusion of pre-cone has the strongest effect on the gyroscopic coupling as observed in Table 1. Large intermodal coupling coefficients between lag and flap appear in the partition matrices' diagonals (i.e., $C_{i, i}^{v, w}=2 \Omega \int_{0}^{L} m \psi_{i}^{v} \psi_{i}^{w} \beta \mathrm{d} x=$ $\left.-C_{i, i}^{w, v}\right)$ due to the Coriolis acceleration. This generates considerable coupling between assumed modes of vibration with 

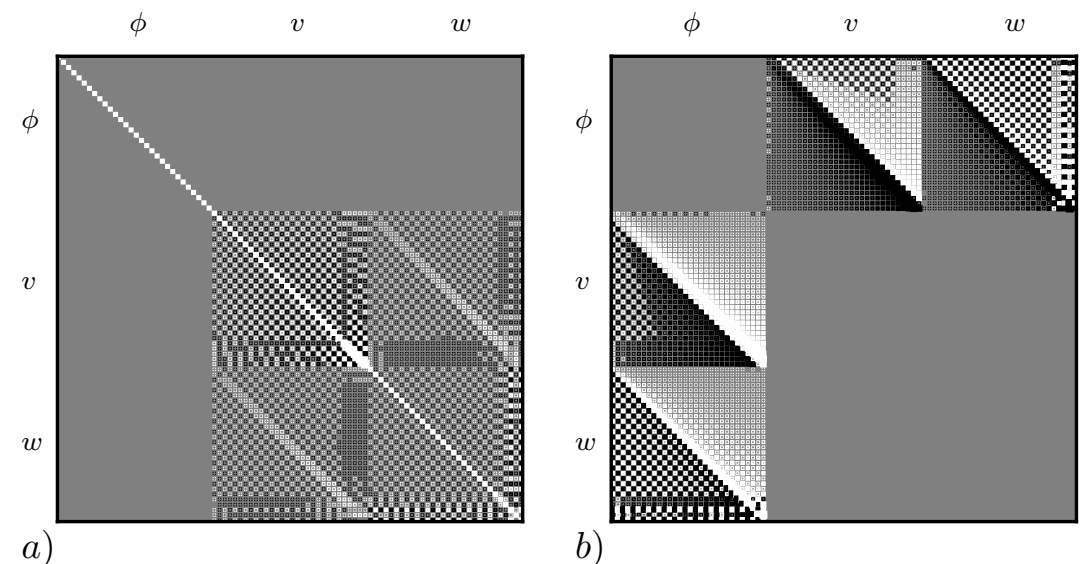

b)

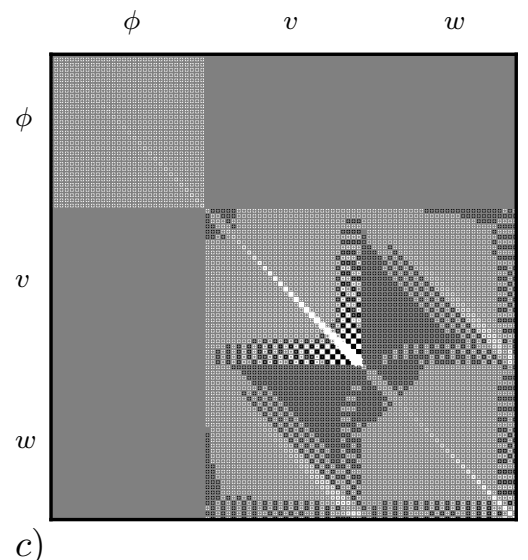

Fig. 12. Hinton diagrams computed for the hingeless rotor blade model described in Ref. $20-\theta^{\prime}=-5$ deg. a) Mass matrix, b) Gyroscopic matrix, c) Overall stiffness matrix.
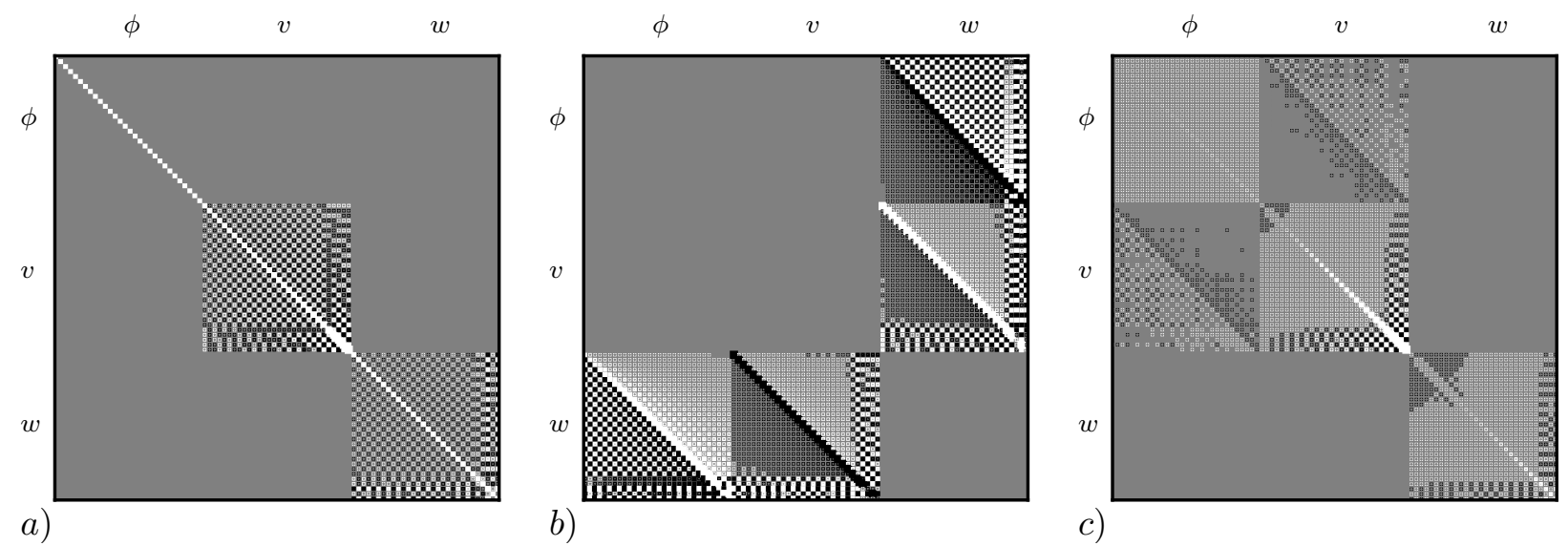

Fig. 13. Hinton diagrams computed for the hingeless rotor blade model described in Ref. $20-\beta=5$ deg. a) Mass matrix, b) Gyroscopic matrix, c) Overall stiffness matrix.

the same mode index $i$. This coupling strengths with the proximity of the flap and lead-lag frequencies in the frequency spectrum. For this particular case of study, the 1st lag and flap modes are closely located. As a result, the effect of the inclusion of the gyroscopic matrix is much larger for these two modes of vibration. Although of smaller magnitude, gyroscopic coupling between flap and torsion does still exist and certain coupling between lag and torsion is observed in the stiffness matrix.

The effect of the inclusion of the gyroscopic matrix in the analysis of modal characteristics has been assessed. Initially, the generation of time-dependent variations on mode shapes has been mathematically demonstrated. Secondly, the relevance of the gyroscopic matrix has been assessed in terms of discrepancies between frequencies obtained from the QEP and GEP for different study cases. The presence of pre-cone has been identified as the key factor in generation of gyroscopic coupling between flap and lag. Therefore, gyroscopic damping needs to be accounted in the modal analysis of rotor blades with large pre-cone angles such as the ones found on tiltrotors. However, it can be omitted without a significant loss of information for conventional helicopter rotors where the pre-cone angle is small. Additionally, the effect of other coupling factors on the coupling between modes of vibration has been assessed, and its magnitude quantified. 


\section{Conclusions}

A mathematical formulation for modelling of coupled flap/lag bending and torsion vibration characteristics of helicopter rotor blades has been presented. The method has been formulated based on the application of Lagrange's equation of motion to the three dimensional kinematics of a non-uniform continuous rotating blade. Spatial solutions of classical theories have been deployed as assumed deformation functions to enhance the computational efficiency of the method. The method has been reduced to a set of closed form integral expressions compatible with different structural boundary conditions. The effect of different geometrical parameters on the coupling between modes of vibration has been assessed. The dependence of the method's prediction on the gyroscopic matrix has been assessed. The predictive vibration characteristics have been compared against experimental measurement andFEA and multi-body dynamics calculations for two hingeless rotors.

Excellent numerical performance based on the use of assumed deformation functions derived from classical methods has been demonstrated. Definite fast convergence with a theoretical infinite number of obtainable modes of vibration has been shown. As a result of the enhanced convergence, converged results are obtained with with the deployment of a low number of assumed deformation functions (Fig. 3). As a consequence, the computational efficiency has been highly improved (Fig. 4). Excellent agreement of the experimental measurements and FEA results with the natural frequencies prediction has been demonstrated for two small scale hingeless rotor blades. Very good agreement between the predicted dominant mode shapes and the experimental measurements has been found, with the exception of the first flap mode. Good agreement between natural frequencies predicted by the proposed method and nonlinear multi-body dynamics calculation has been observed for a small scale rotor blade. Additionally, excellent agreement between the predicted dominant mode shapes and multi-body calculations has been identified for the small scale hingeless rotor. The effect of the gyroscopic matrix on the vibration predictions has been assessed. Time-dependent variations in mode shapes have been associated with the inclusion of the gyroscopic effect in the analysis. Pre-cone has been identified as the key parameter in the generation of gyroscopic coupling between chordwise and flapwise displacements.

The proposed method has been demonstrated to accurately estimate the first 7 modes of vibration a fraction of a second. Its computational efficiency and the very limited required information present this method as an efficient alternative to FEA or multi-body dynamics for the structural analysis of rotor blades during the preliminary design phase. Its further integration into a flight dynamics code acts as an enabler for the generation of comprehensive codes for the analysis of novel rotorcraft configuration where strong coupling between modes of vibration is present.

\section{References}

[1] Timoshenko SP. On the Correction for Shear of the Differential Equation for Transverse Vibrations of Prismatic Bars. The London, Edinburgh, and Dublin Philosophical Magazine and Journal of Science. 1921 May;41(245):744-746.

[2] Vlasov VZ. Thin-Walled Elastic Beams. National Science Foundation and Department of Commerce; 1961.

[3] Houbolt JC, Brooks GW. Differential Equations of Motion For Combined Flapwise Bending, Chordwise Bending, and Torsion of Twisted Nonuniform Rotor Blades. NACA; 1957. NACA/TN-3905.

[4] Hodges DH, Dowel EH. Nonlinear Equations of Motion for the Elastic Bending and Torsion of Twisted Nonuniform Rotor Blades. NASA; 1974. NASA/TN-7818.

[5] Hodges DH, Ormiston RA. Stability of Elastic Bending and Torsion of Uniform Cantilever Rotor Blades in Hover with Variable Structural Coupling. NASA; 1976. NASA/TN-8192.

[6] Hodges DH, Ormiston RA, Peters DA. On the Nonlinear Deformation Geometry of Euler-Bernoulli Beams. NASA; 1980. NASA/TP-1566.

[7] Hodges DH. Nonlinear Equations for Dynamics of Pretwisted Beams Undergoing Small Strains and Large Rotations. NASA; 1985. NASA/TP-2470.

[8] Berdichevskii VL. Variational-Asymptotic Method of Constructing the Nonlinear Shell Theory. Theory of shells. 1980 July;p. $137-161$.

[9] Hodges DH, Atilgan AR, Cesnik CES, Fulton MV. On a Simplified Strain Energy Function for Geometrically Nonlinear Behaviour of 
Anisotropic Beams. Composites Engineering. 1992 December;2(5):513-526.

[10] Hodges DH, Lee BW, Atilgan AR. Application of the Variational-Asymptotical Method to Laminated Composite Plates. AIAA journal. 1993 September;31(9):1674-1683.

[11] Murthy V. Dynamic Characteristics of Rotor Blades. Journal of Sound and Vibration. 1976;49(4):483-500.

[12] Surace G, Anghel V, Mares C. Coupled Bending-Bending-Torsion Vibration Analysis of Rotating Pretwisted Blades: an Integral Formulation and Numerical Examples. Journal of Sound and Vibration. 1997;206(4):473-486.

[13] Fasana A, Marchesiello S. Rayleigh-Ritz Analysis of Sandwich Beams. Journal of sound and vibration. 2001 April;241(4):643-652.

[14] Hodges DH. Vibration and Response of Nonuniform Rotating Beams with Discontinuities. Journal of the American Helicopter Society. 1979 October;24(4):43-50.

[15] Goulos I, Pachidis V, Pilidis P. Lagrangian Formulation for the Rapid Estimation of Helicopter Rotor Blade Vibration Characteristics. Aeronautical Journal. 2014 August;118(1206):861-901.

[16] Goulos I, Pachidis V, Pilidis P. Helicopter Rotor Blade Flexibility Simulation for Aeroelasticity and Flight Dynamics Applications. Journal of the American Helicopter Society. 2014 October;59(4):1-18.

[17] Goulos I, Pachidis V, Pilidis P. Flexible Rotor Blade Dynamics for Helicopter Aeromechanics Including Comparisons with Experimental Data. Aeronaut J. 2014 December;119(1213):301-342.

[18] Goulos I, Pachidis V. Real-Time Aero-Elasticity Simulation of Open Rotors With Slender Blades for the Multidisciplinary Design of Rotorcraft. Journal of Engineering for Gas Turbines and Power. 2015 January;137(1):012503.

[19] Tisseur F, Meerbergen K. The Quadratic Eigenvalue Problem. SIAM Review. 2001 June;43(2):235-286.

[20] Sharpe DL. An Experimental Investigation of the Flap-Lag-Torsion Aeroelastic Stability of a Small-Scale Hingeless Helicopter Rotor in Hover. NASA; 1986. NASA/TP-2546.

[21] Jung SN, You YH, Dhadwal MK, Hagerty BP, Riemenschneider J, Keimer R. Blade Property Measurement and Its Assessment on Air/Structural Loads of HART II Rotor. In: Proceedings of the 70th Annual Forum. American Helicopter Society; 2014. .

[22] Srinivasan AV, Cutts DG, Shu HT, Sharpe DL, Bauchau OA. Structural Dynamics of a Helicopter Rotor Blade System. Journal of The American Helicopter Society. 1990 May;35(1):75-85.

[23] Furchert R. Bestimmung der Eigenfrequenzen und Eigenformen von 3 Rotorblättsa des Rotorversuchsstands (Determination of Natural Frequencies and Mode Shapes of 3 Rotor Blade Sets of the RotorTest Rig). DLR; 1992. DLR-IB 111-292/11.

[24] van der Wall BG. 2nd HHC Aeroacoustic Rotor Test (HART II)-Part I: Test Documentation. DLR; 2003. DLR-IB 111-2003/31.

[25] van der Wall BG, Lim JW, Smith MJ, Jung SN, Bailly J, Baeder JD, et al. The HART II International Workshop: an Assessment of the State-of-the-Art in Comprehensive Code Prediction. CEAS Aeronautical Journal. 2013 September;4(3):223-252.

\section{A. Nomenclature}

Roman Symbols
$[Q(\lambda)]$
$\{\bar{q}\}_{i}$
$\tilde{e}_{x}, \tilde{e}_{y}, \tilde{e}_{z}$
$\tilde{r}$
$\tilde{V}$
$A(x)$
$B_{1}^{*}(x), B_{2}^{*}(x)$
$C_{i, j}^{r, s}$

dynamic system resultant $\lambda$-Matrix

$i$ th eigenvector of $\lambda$-Matrix $[Q(\lambda)]$

unit vectors of the orthogonal coordinate system with origin at the blade hinge/root, $X$ along the undeformed blade and $Y$ towards the leading edge, $\mathrm{m}$

position vector of a point $\mathrm{P}$ relative to the blade root/hinge offset , $\mathrm{m}$

Velocity vector of a point $\mathrm{P}$ relative to the blade root/hinge offset, $\mathrm{m} \mathrm{s}^{-1}$

cross-sectional area, $\mathrm{m}^{2}$

blade cross-sectional integrals, $\mathrm{m}^{6}, \mathrm{~m}^{5}$

gyroscopic intermodal coupling coefficient between the $i$ th and $j$ th assumed deformation functions of the $r$ and $s$ DOFs 


$e$
$E(x), G(x)$
$e_{A}(x), e_{M}(x)$
$F_{i, j}^{r, s}$
$f_{i}^{r}$
$G_{i, j}^{r, s}$
$I_{1}(x), I_{2}(x)$
$I_{p}(x)$
$I_{y}(x), I_{z}(x)$
$J(x)$
$K^{r}$
$L$
$m^{r}(x)$
$M_{i, j}^{r, s}$
$N$
$Q_{i}^{r}$
$q_{i}^{r}(t)$
$R$
$S_{i, j}^{r, s}$
$t$
$T, U$
$u(x, t)$
$v(x, t)$
$w(x, t)$
$X_{i}^{r}(x)$
$x_{T}(x)$
${ }^{r}$

Greek Symbols

$\beta$

$\epsilon_{x x}, \epsilon_{x \eta}, \epsilon_{x \zeta}$

$\eta, \zeta$

$\lambda$

$\left\{\Psi^{r}(x)\right\}$

$\Omega$

$\phi(x, t)$

$\psi_{i}^{r}(x)$

$\rho$

$\theta(x)$

$\tilde{\Omega}$

\section{Superscripts}

blade root/hinge offset from the hub center as a fraction of rotor radius

material Young's, shear stress moduli, $\mathrm{Pa}$

area centroid, mass centroid offsets from the blade elastic axis, $\mathrm{m}$

centrifugal stiffening intermodal coupling coefficient between the $i$ th and $j$ th assumed deformation functions of the $r$ and $s$ DOFs

centrifugal static force coefficient for the $i$ th assumed deformation function of the $r$ DOF

elastic intermodal coupling coefficient between the $i$ th and $j$ th assumed deformation functions of the $r$ and $s$ DOFs

blade cross-sectional mass moments of inertia about major, minor axes, $\mathrm{kg} \mathrm{m}$

blade cross-sectional mass polar moment of inertia, $\mathrm{kg} \mathrm{m}$

blade cross-sectional moments of inertia about major, minor axes, $\mathrm{m}^{4}$

blade cross-sectional polar moment of inertia, $\mathrm{m}^{4}$

hinge/pitch-control system spring stiffness of the $r$ DOF, $\mathrm{N} \mathrm{m} \mathrm{rad}^{-1}$

actual blade length, $=R(1-e), \mathrm{m}$

blade mass per unit length, $\mathrm{kg} \mathrm{m}^{-1}$

mass intermodal coupling coefficient between the $i$ th and $j$ th assumed deformation functions of the $r$ and $s$ DOFs

number of assumed deformation functions per DOF

generalized force corresponding to the $i$ th generalized coordinate of the $r$ DOF

time-dependent generalized coordinate of the $i$ th assumed deformation function of the $r$ DOF

rotor blade radius, $\mathrm{m}$

overall stiffness intermodal coupling coefficient between the $i$ th and $j$ th assumed deformation functions

of the $r$ and $s$ DOFs

time, $\mathrm{s}$

kinetic, potential energies of the rotor blade, $\mathrm{J}$

axial ( $X$ axis) time-dependent beam element displacement, $\mathrm{m}$

chordwise ( $Y$ axis)time-dependent beam element displacement, $\mathrm{m}$

flapwise ( $Z$ axis) time-dependent beam element displacement, $m$

shape for the $i$ th mode of motion of the $r$ DOF, m, rad

beam element centrifugal displacement $\left(x\right.$ axis), $\int_{0}^{x} \frac{\int_{\lambda}^{L} m \Omega^{2}(\varrho+e R) \mathrm{d} \varrho}{E A} \mathrm{~d} \lambda, \mathrm{m}$

pre-cone angle, rad

classical strain components

cross-sectional coordinates along the major, minor principal axes, $\mathrm{m}$

eigenvalue of the $\lambda$-Matrix $[Q(\lambda)], \operatorname{rad~s}^{-1}$

assumed deformation function vector of the $r \mathrm{DOF},=\left[\psi_{j}^{r}(x), j=1, \ldots N\right]^{T}$

blade rotorspeed, $\operatorname{rad~s}^{-1}$

time-dependent torsional deformation angle, rad

assumed deformation function of the $i$ th mode shape of the $r$ DOF, $\mathrm{m}, \mathrm{rad}$

material density, $\mathrm{kg} \mathrm{m}^{-3}$

cross-sectional pretwist angle, rad

blade rotorspeed vector, $\operatorname{rad~s}^{-1}$

1st and 2nd derivatives with respect to time $t$ 
$\begin{array}{ll}()^{\phi, v, w} & \text { referring to torsion, lag or flap respectively } \\ ()^{\prime},()^{\prime \prime} & \text { 1st and 2nd derivatives with respect to axial coordinate } x \\ ()^{r, s} & \text { DOF indices }\end{array}$

\section{Subscripts}

()$_{0} \quad$ referring to the root/hinge location

()$_{i, j} \quad$ mode number indices

Acronyms

DOF

degree of freedom

FEA

finite element analysis

GEP

generalized eigenvalue problem

QEP

quadratic eigenvalue problem 
2016-11-13

\title{
Modelling and analysis of coupled flap-lag-torsion vibration characteristics helicopter rotor blades
}

\author{
Castillo Pardo, Alejandro
}

Sage Publications

Castillo Pardo A, Goulos I, Pachidis V. (2017) Modelling and analysis of coupled flap-lag-torsion vibration characteristics helicopter rotor blades. Proceedings of the Institution of Mechanical

Engineers, Part G: Journal of Aerospace Engineering, Volume 231, Issue 10, 2017, pp. 1804-1823 https://dspace.lib.cranfield.ac.uk/handle/1826/10979

Downloaded from Cranfield Library Services E-Repository 